\title{
Exploring large charge density systems with lattice QCD+QED
}

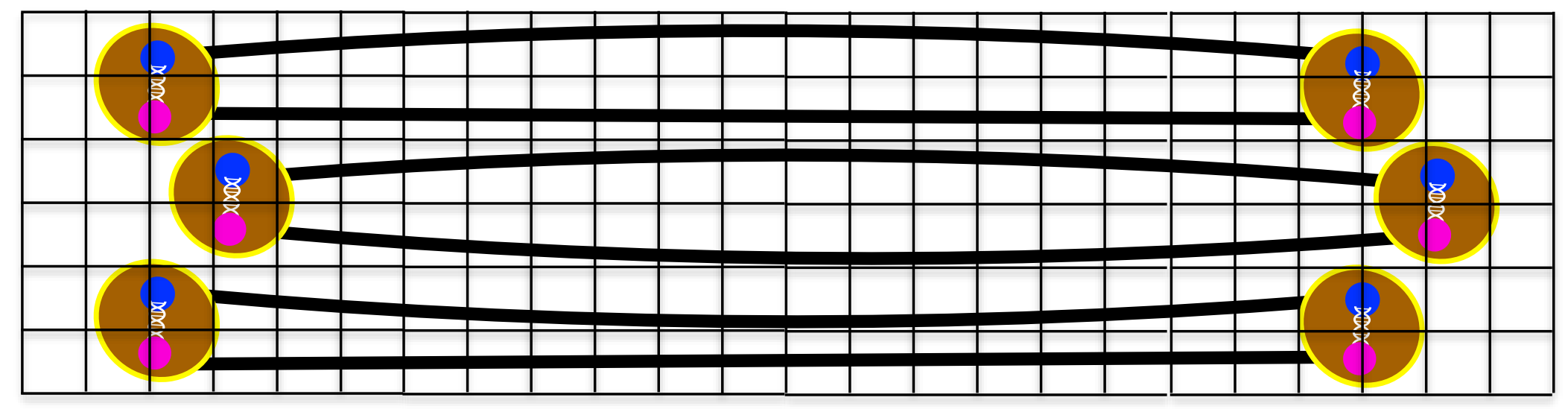

Michael Wagman

\section{University of Edinburgh Particle Physics Theory Seminar}

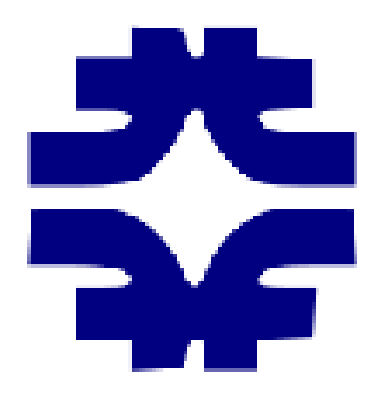
Fermilab

\section{May 5, 2021}

Beane et al, PRD 103 (2021) arXiv:2003.12130

in collaboration with Beane, Detmold, Horsley, Illa, Jafry, Murphy, Nakamura,

Perlt, Rakow, Schierholz, Shanahan, Stüben, Winter, Young, and Zanotti 


\section{Precision theory and experiment}

Precise deviations between Standard Model theory and experiment could be our best probes of physics beyond the Standard Model

New physics searches sensitive to lowenergy hadronic physics require nonperturbative QCD input

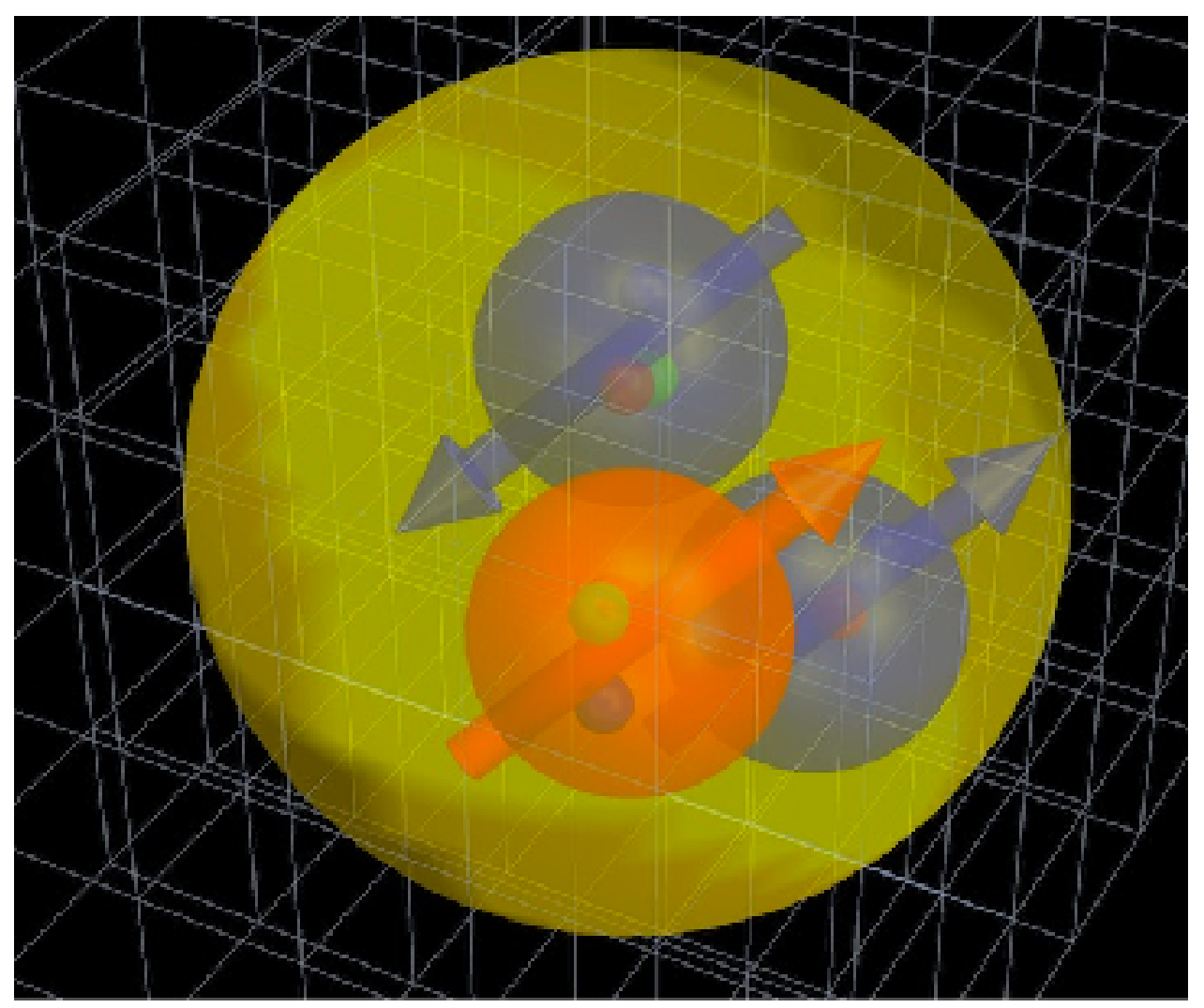

W. Detmold

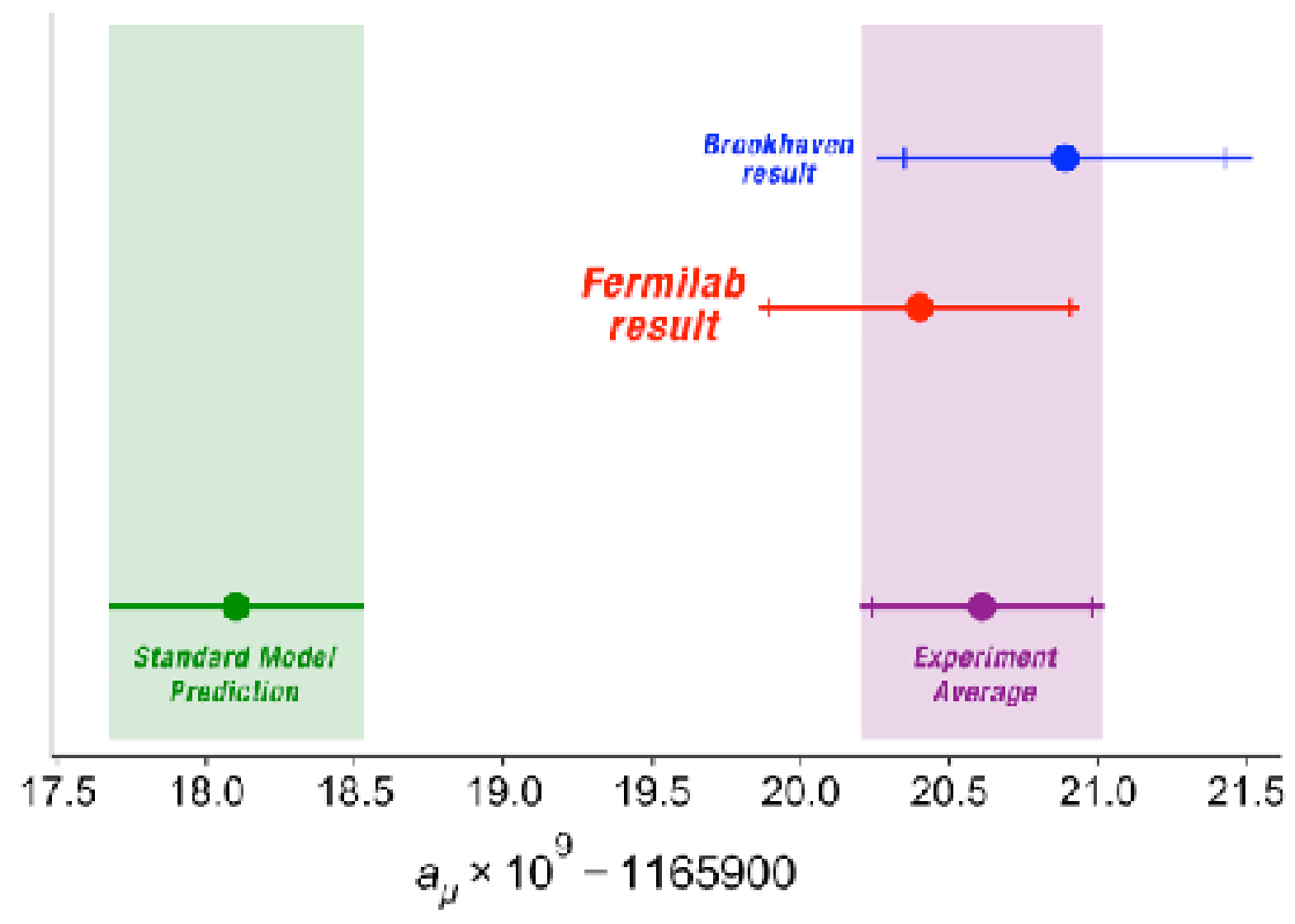

QCD path integrals turned into large but finite dimensional integrals by discretizing spacetime (UV regulator) and working in a finite volume (IR regulator)

Monte Carlo methods enable calculations on large but finite computers 


\section{Lattice QCD+QED}

High precision calculations of physical quantities require including dynamical QED effects nonperturbatively in lattice QCD calculations

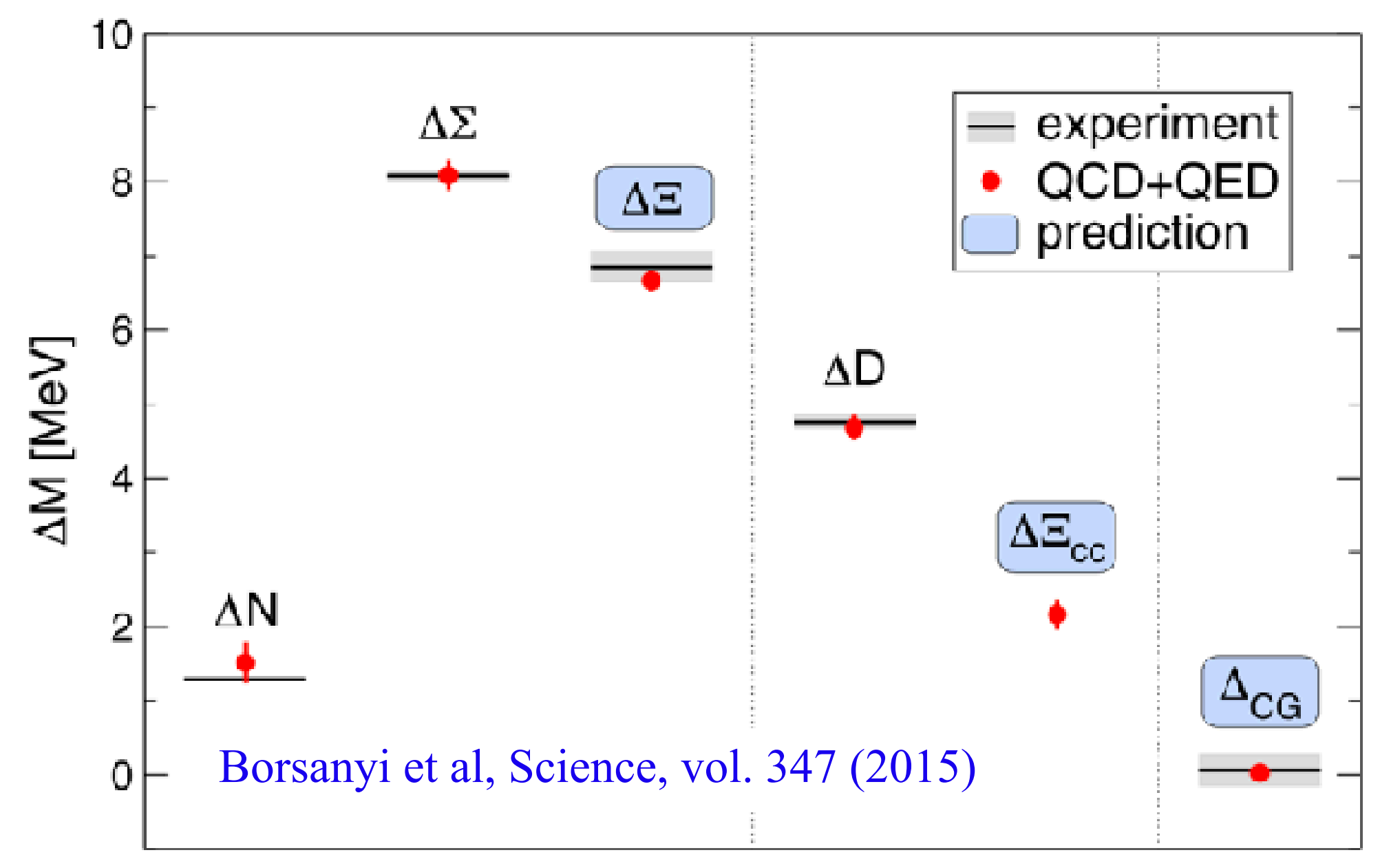

Precise LQCD+QED determinations of meson and baryon masses have reproduced experimental results and made new predictions

Extending precise LQCD+QED results to multi-hadron systems including nuclei faces both numerical and theoretical challenges 


\section{New physics and nuclei}

Nuclei are abundant and useful experimental targets

Converting between nuclear- and nucleon-level cross-sections requires

- Nuclear models (error bar ?s)

- Direct LQCD calculations (impractical)

- LQCD informed EFT + modeling

Xenon1T constraint on dark matter-nucleus

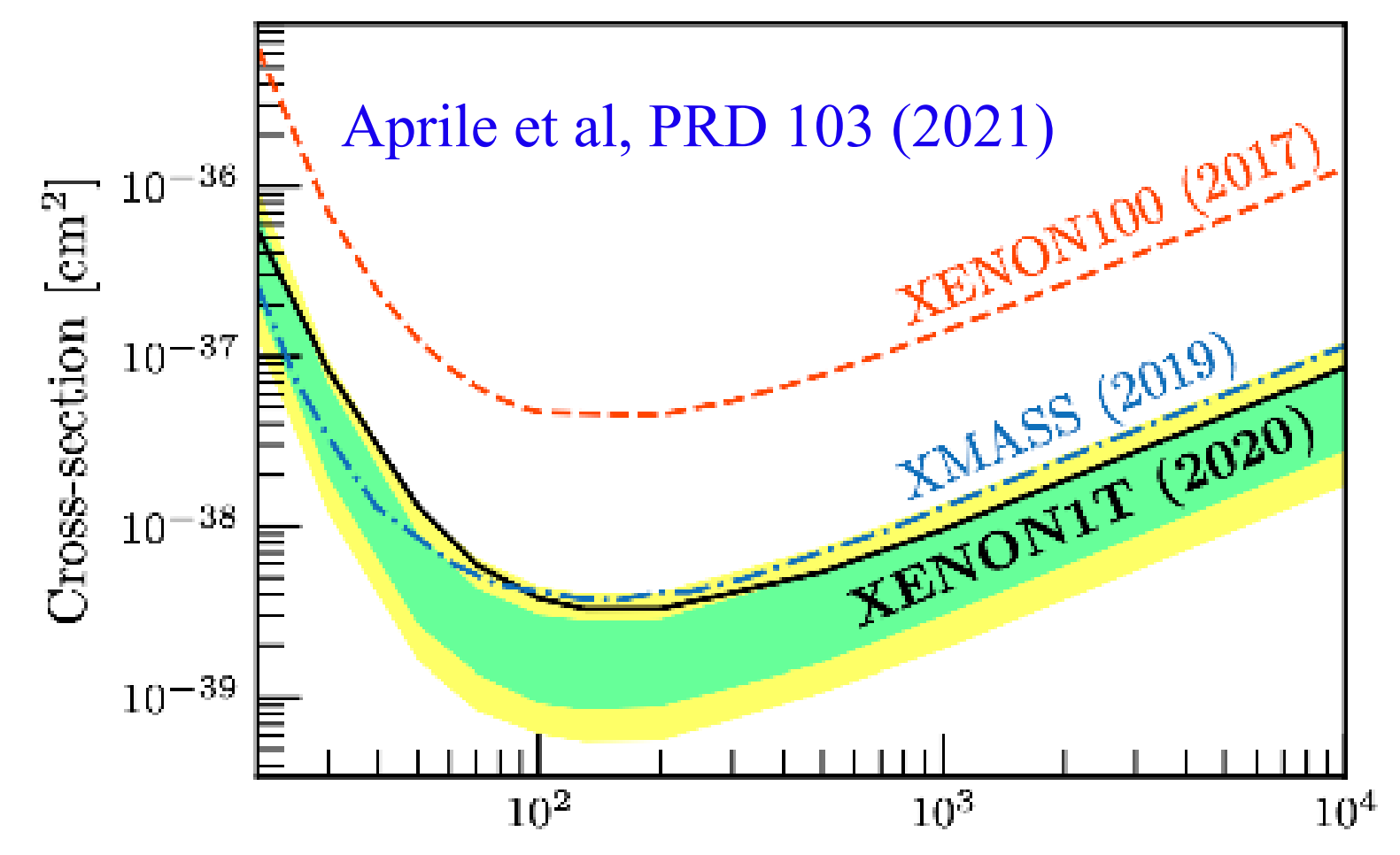

Next-generation accelerator neutrino experiments require

WIMP mass $\left[\mathrm{GeV} / \mathrm{c}^{2}\right]$

few-percent level control of nuclear cross-sections

Abi et al, DUNE Technical Design Report arXiv:2002.03005

Standard Model predictions with controlled uncertainties essential

DUNE

\section{Santord}

Underground

Research

Facility

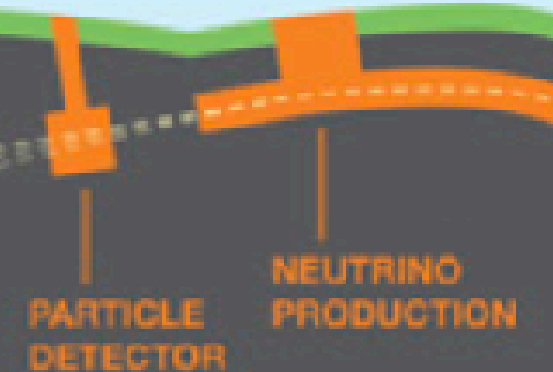

Fermilab PARTICLE 


\section{Nuclei and lattice QCD}

Exploratory calculations performed of nuclear matrix elements relevant for electroweak and beyond-Standard-Model processes

Review, Davoudi, MW et al, Phys.Rept. 900 (2021)

Exponential signal-to-noise degradation limits LQCD calculations to lightest nuclei, and so far to unphysically heavy quark masses

Triton beta-decay

Systematic uncertainties not yet fully controlled (one lattice spacing, heavy quark masses, ...)

Results provide proof-of-principle that LQCD can determine properties and interactions of multi-nucleon systems

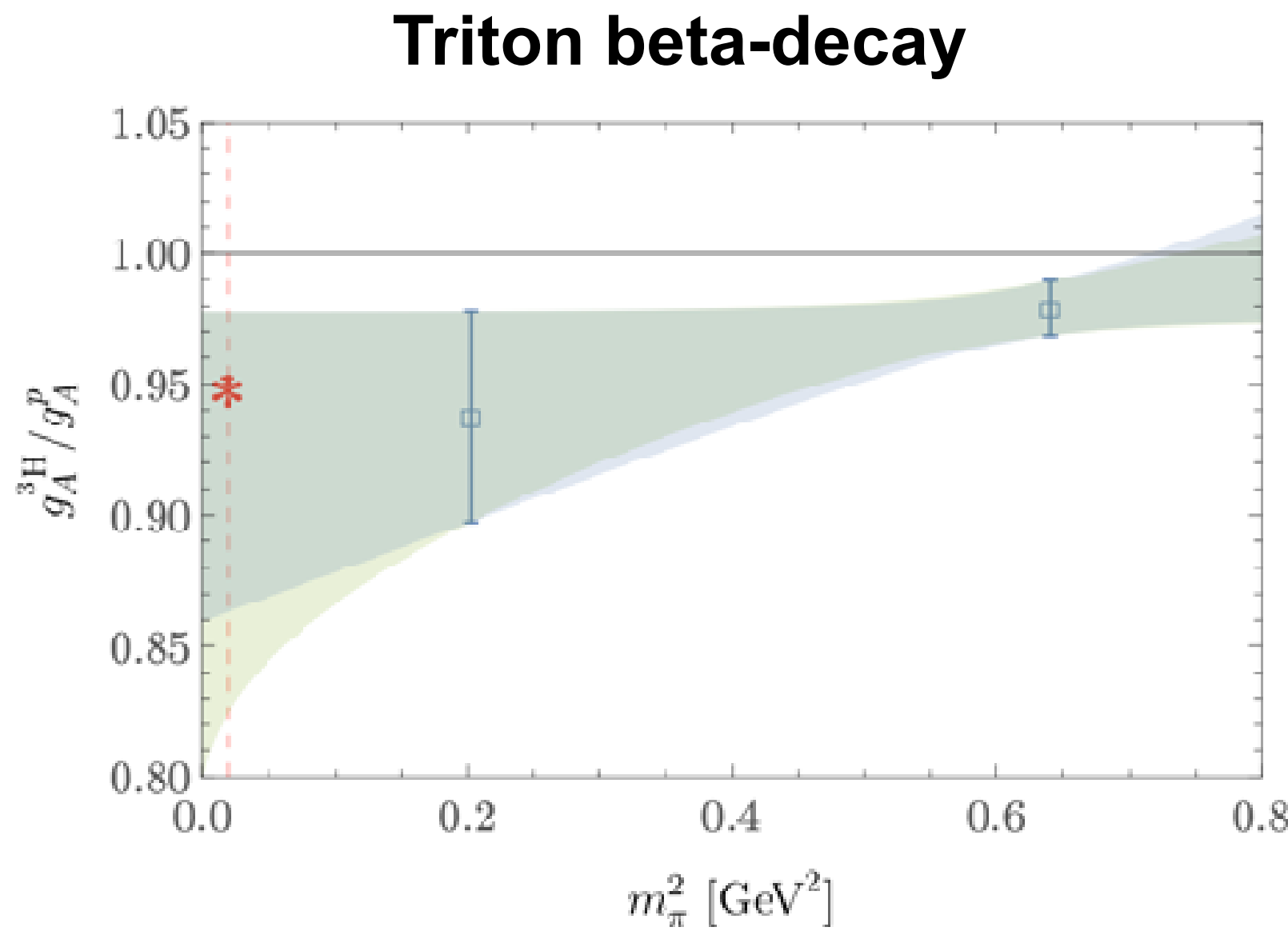




\section{QCD+QED phenomenology}

\section{Charged hadron scattering}

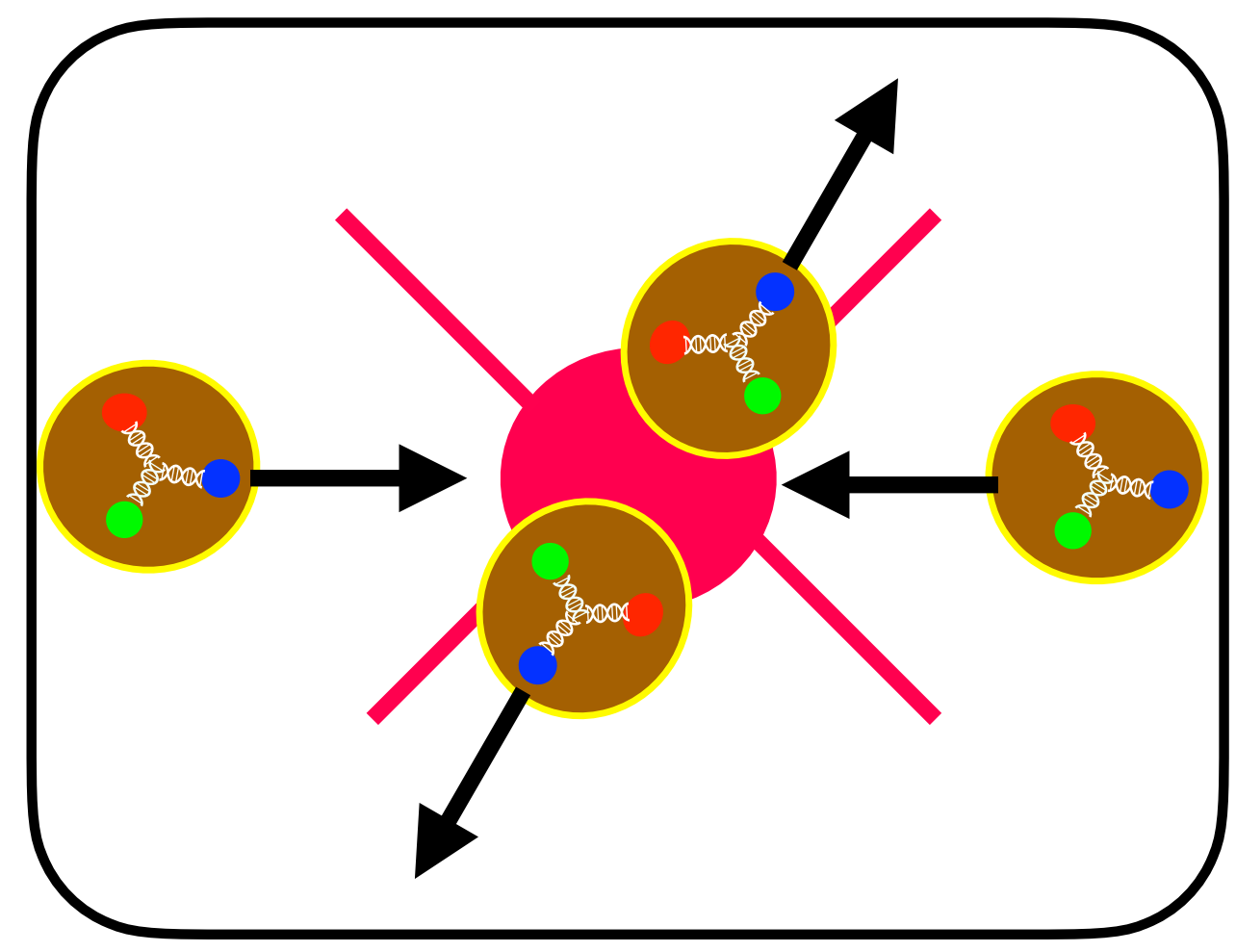

LQCD+QED can separate strong and electromagnetic isospin violation in nucleon-nucleon scattering lengths

Precise predictions of isospin-violating differences between $p p, p n$, and $n n$ scattering lengths would improve nuclear EFTs

\section{High charge-density systems}

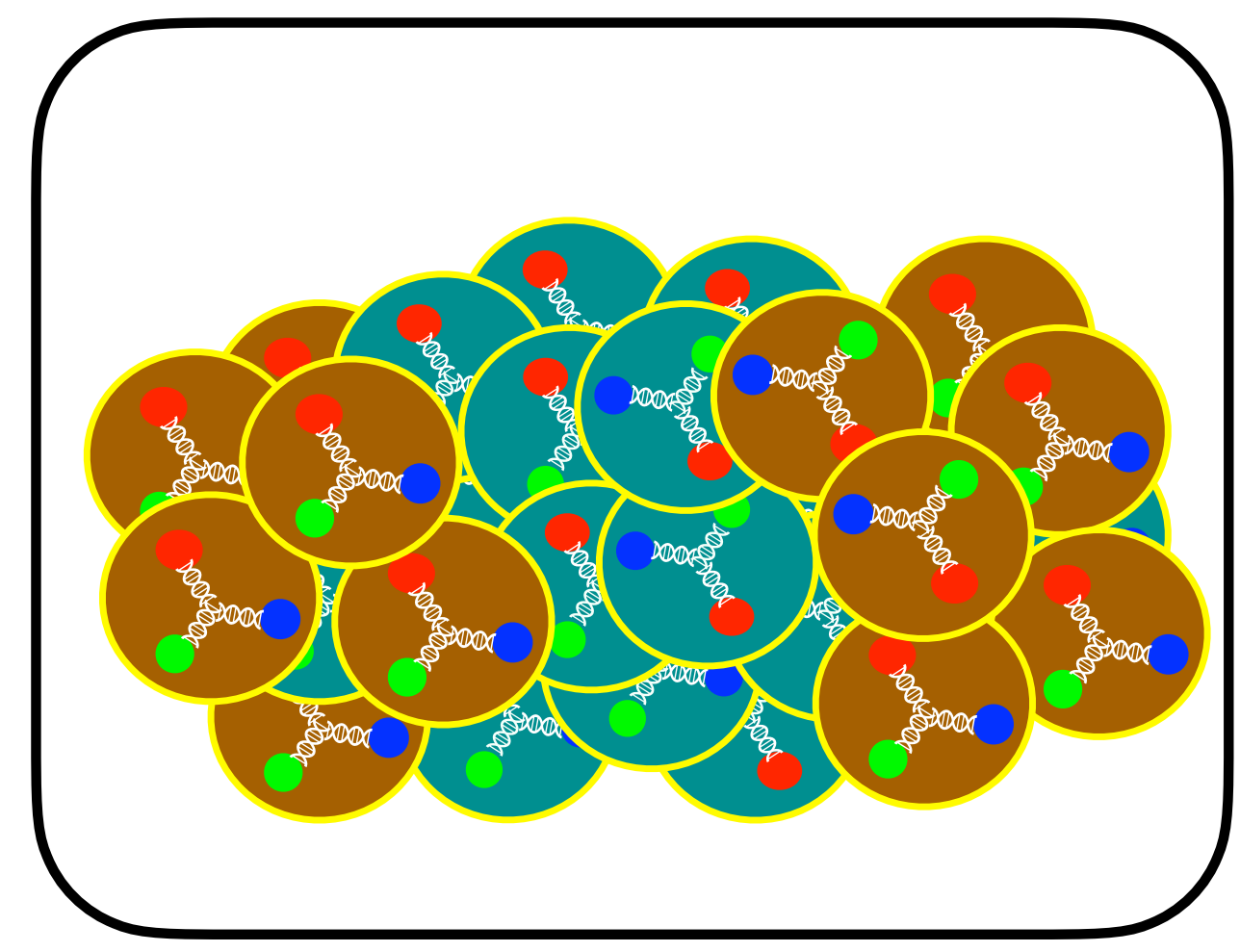

Boundaries of periodic table determined by competition between QCD and QED

LQCD+QED calculations in principle can disentangle nonperturbative QED and QCD effects in nuclei 


\section{QCD+QED in a box}

LQCD+QED for $\alpha Z>1$ nuclei faces several obstacles:

Charged systems in a box with periodic boundary conditions violate Gauss's law

- Either boundary conditions or photon field must be modified

Review: Patella, PoS LATTICE 2016 (2017)

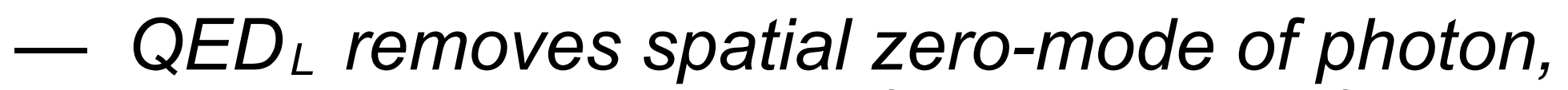
equivalent to adding uniform density of opposite charge

Duncan et al, PRL 76 (1996)

Blum et al, PRD 76 (2007)

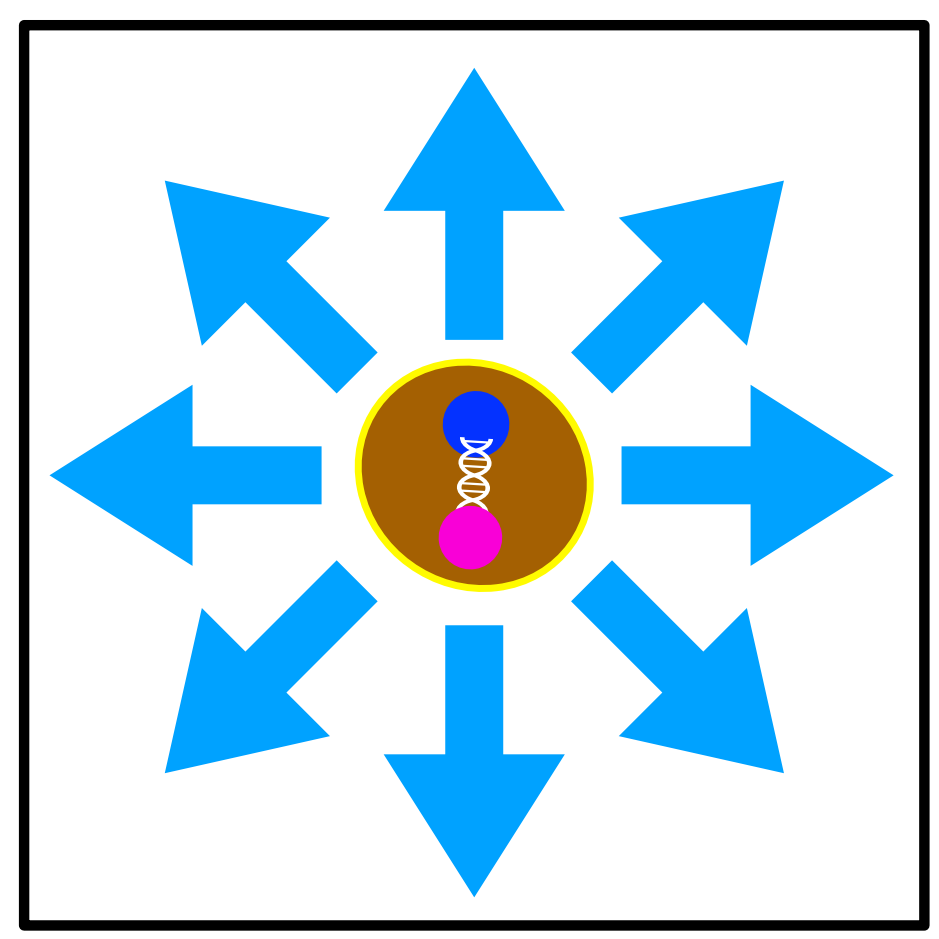

Hayakawa and Uno, Prog. Theor. Phys. 120 (2008)

QCD+QED $D_{L}$ preserves symmetries of QCD+QED but violates locality

- Subtleties of nonlocal field theory must be understood in order to match $L Q C D+Q E D_{L}$ results to hadronic EFTs

Exponential signal-to-noise degradation with increasing baryon number

- Not this talk. Start with $\angle Q C D+Q E D_{L}$ for charged mesons with unphysically large $\alpha$ to explore high charge density systems at lower cost 


\section{A charged particle in a box}

A charged particle system in a finite volume (FV) requires care to define

- Not gauge invariant - must gauge fix to obtain non-zero correlation functions

- Violates Gauss's law — remove zero mode

$$
A_{\mu}(x)=\int \frac{d p^{0}}{2 \pi} \frac{1}{L^{3}} \sum_{\mathbf{n} \in \mathbb{Z}^{3} \backslash\{\mathbf{0}\}} e^{-i p^{0} x^{0}+\frac{2 \pi i}{L} \mathbf{n} \cdot \mathbf{x}} \tilde{A}_{\mu}\left(p^{0}, \mathbf{n}\right)
$$

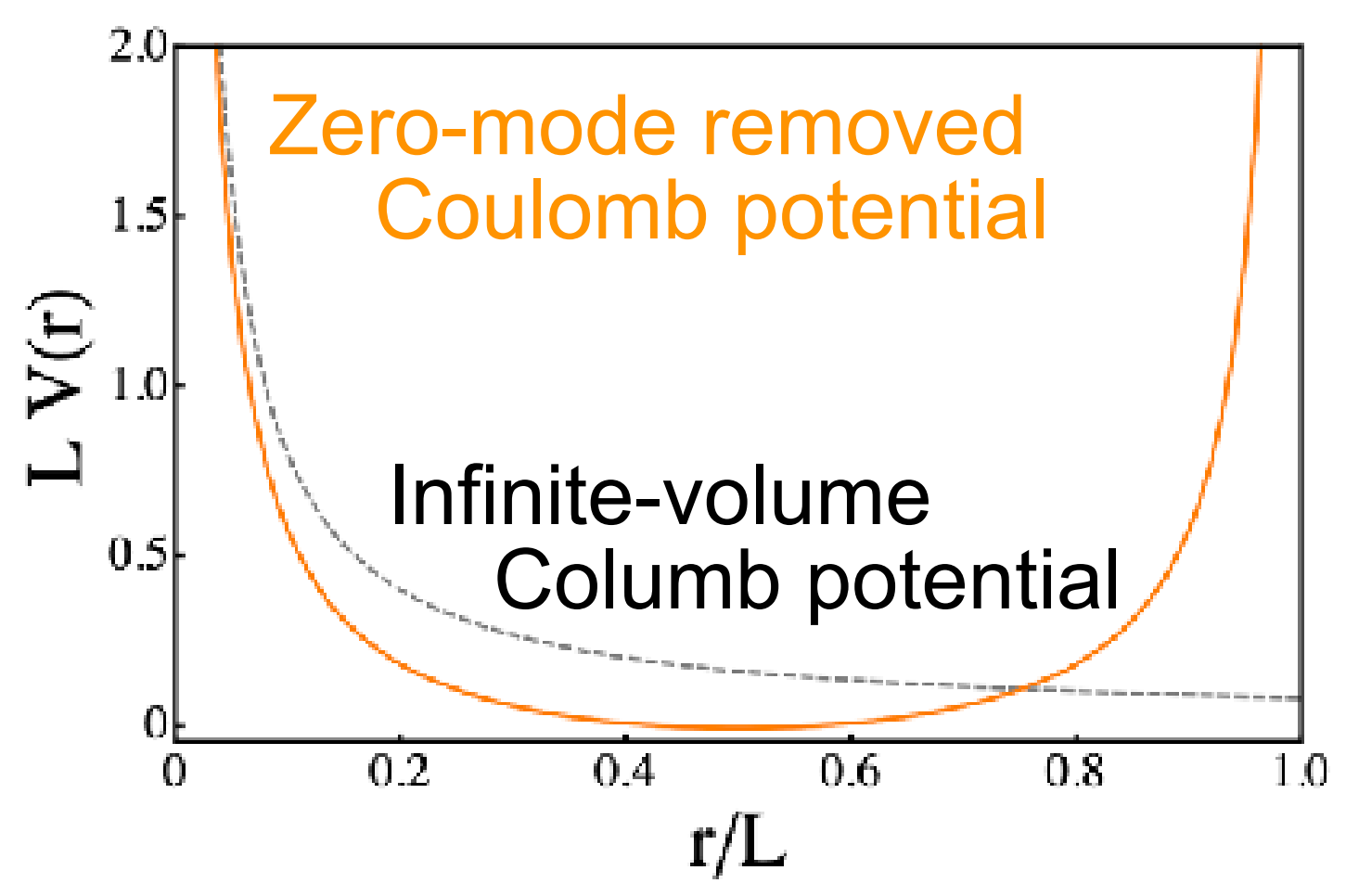

Quantized momenta $\mathbf{p}=\frac{2 \pi}{L} \mathbf{n}$

Davoudi and Savage, PRD 90 (2014)

Massless photon exchange leads to power law FV effects different from exponentially suppressed FV effects associated with massive particles
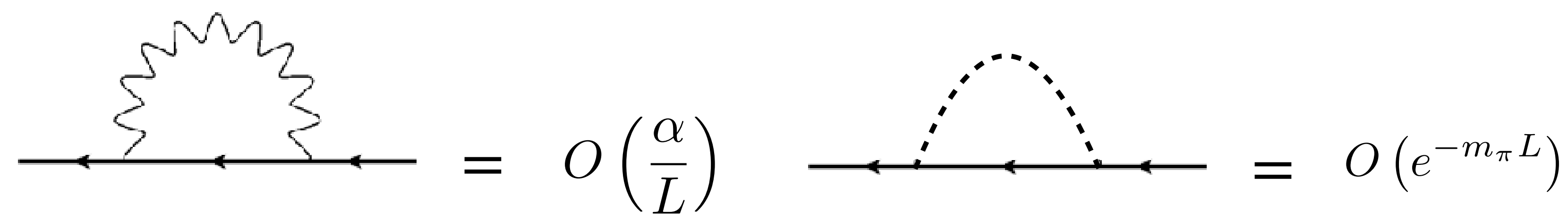


\section{Two particles in a box}

Volume-dependence of twoparticle states encodes scattering phase shifts

Huang, Yang, Phys. Rev. 105 (1957)

Lüscher, Commun. Math. Phys. 105 (1986)
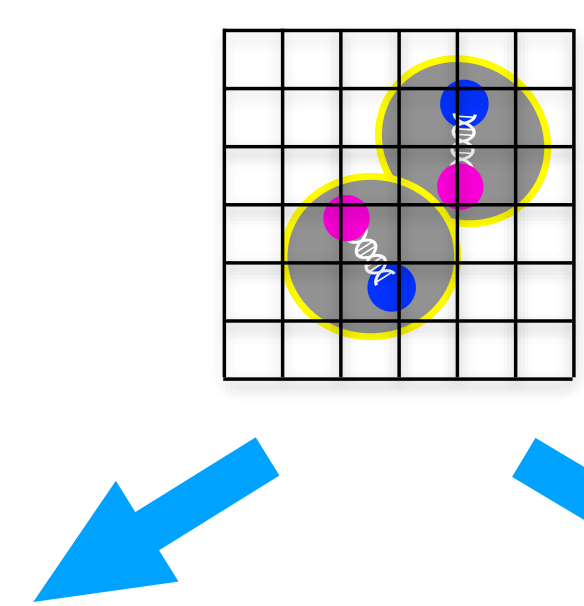

Infinite-volume bound state

$$
[E(L)-E(\infty)] \propto \frac{e^{-\gamma L}}{\gamma L}
$$

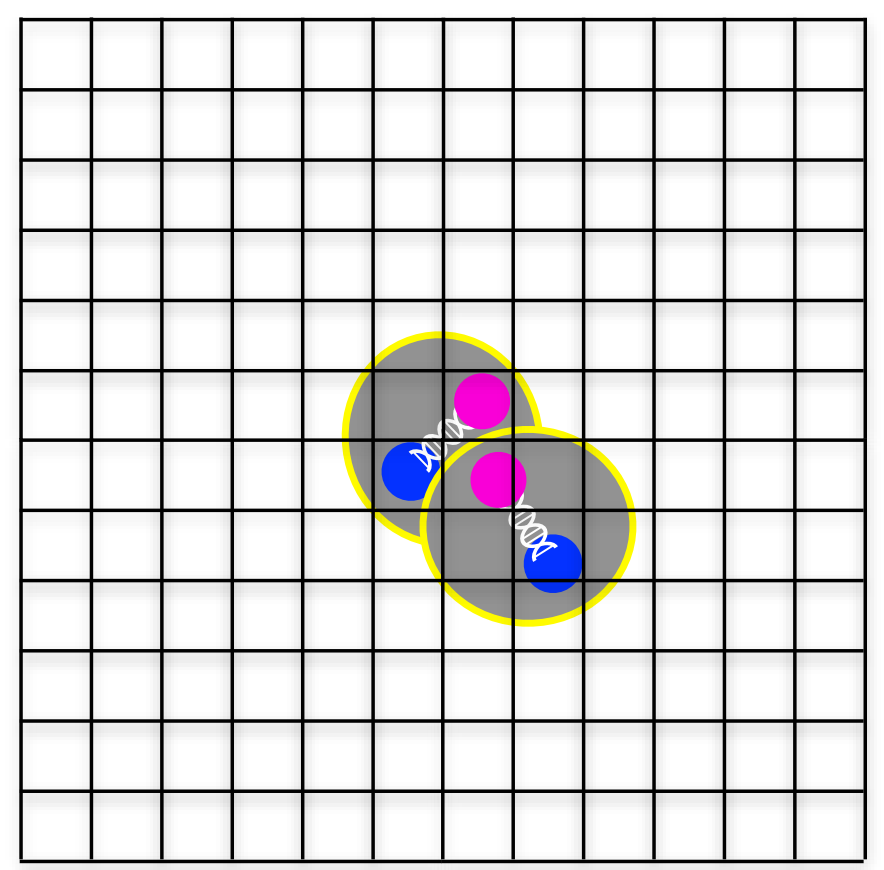

Lüscher's quantization condition and generalizations now used to study a wide range of hadronic resonances

Review: Briceño, Dudek, Young, Rev. Mod. Phys. 90 (2018)
Infinite-volume scattering state

$$
[E(L)-E(\infty)] \propto \frac{a}{M L^{3}}
$$

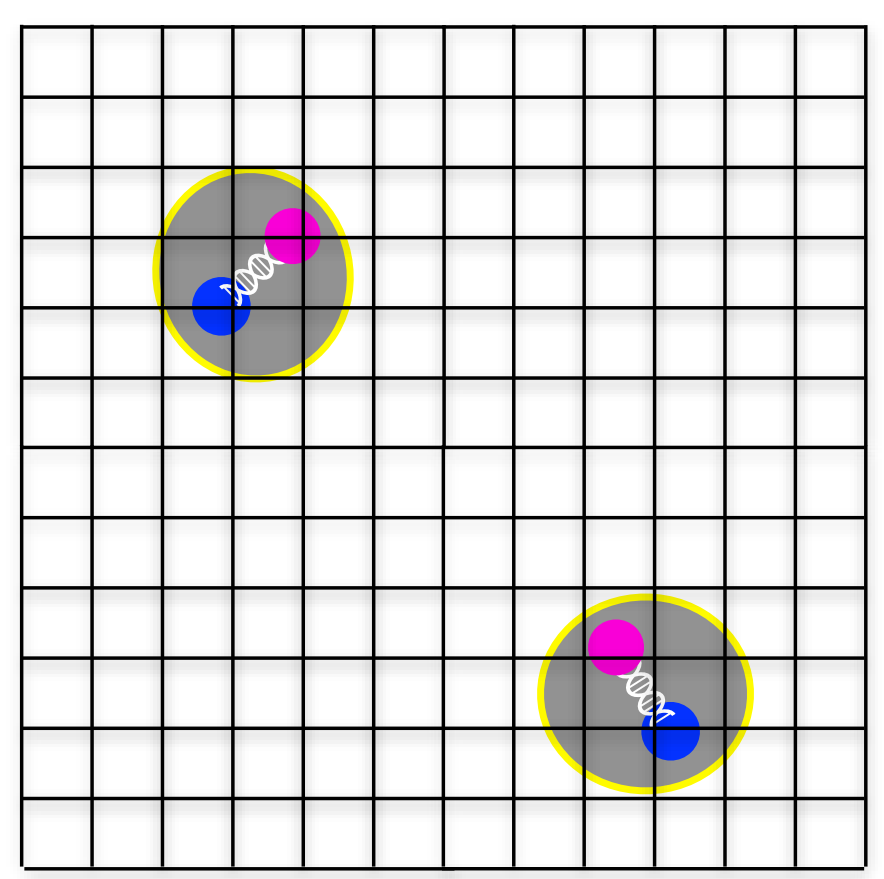




\section{NRQED}

Significant recent progress in deriving three-particle quantization conditions

Review: Hansen and Sharpe, Ann. Rev. Nucl. Part. Sci. 69 (2019)

Relativistic formalism for FV effects on generic particle-number systems unknown

Non-Relativistic QED (NRQED) describes low-energy QED for electromagnetic bound states, charged particle scattering, hadronic reactions and decays, ...

Caswell and Lepage, Phys. Lett. 167B (1985)

Kong and Ravndal, Nucl. Phys. A 665 (2000)

Carrasco et al, PRD 91 (2015)

\section{Dual expansion:}

$\alpha$

$$
p / M
$$

Power counting (on-shell matter particles):
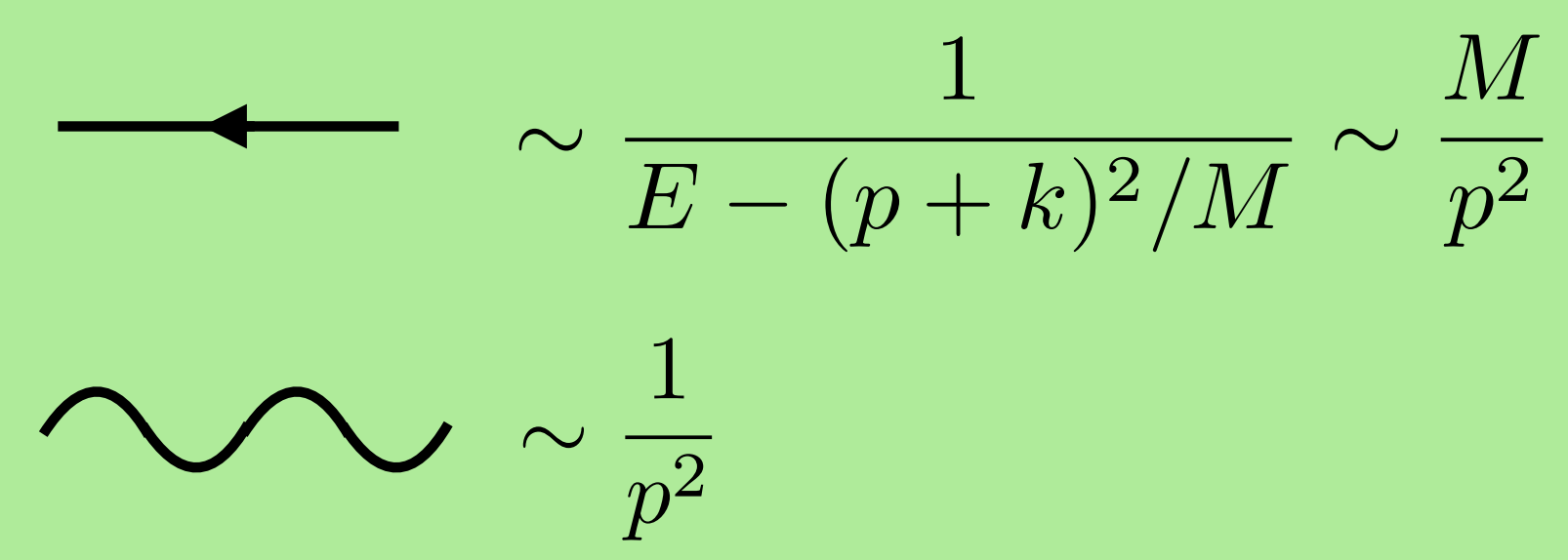

$\int d E \int d^{3} k \sim \frac{p^{5}}{M}$ 


\section{Nonperturbative Coulomb}

With multiple charged particles, loop expansion does not correspond to EFT expansion

Coulomb ladder diagrams must be resumed for

$$
\eta=\frac{\alpha M}{2 p}>1
$$

Applying power counting:

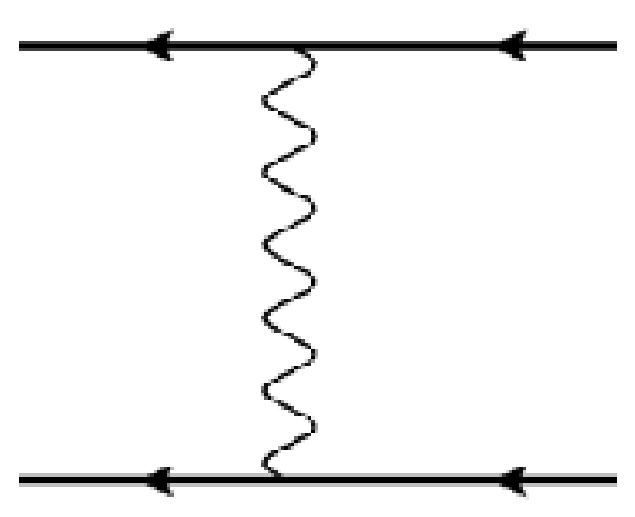

Power counting (on-shell matter particles):
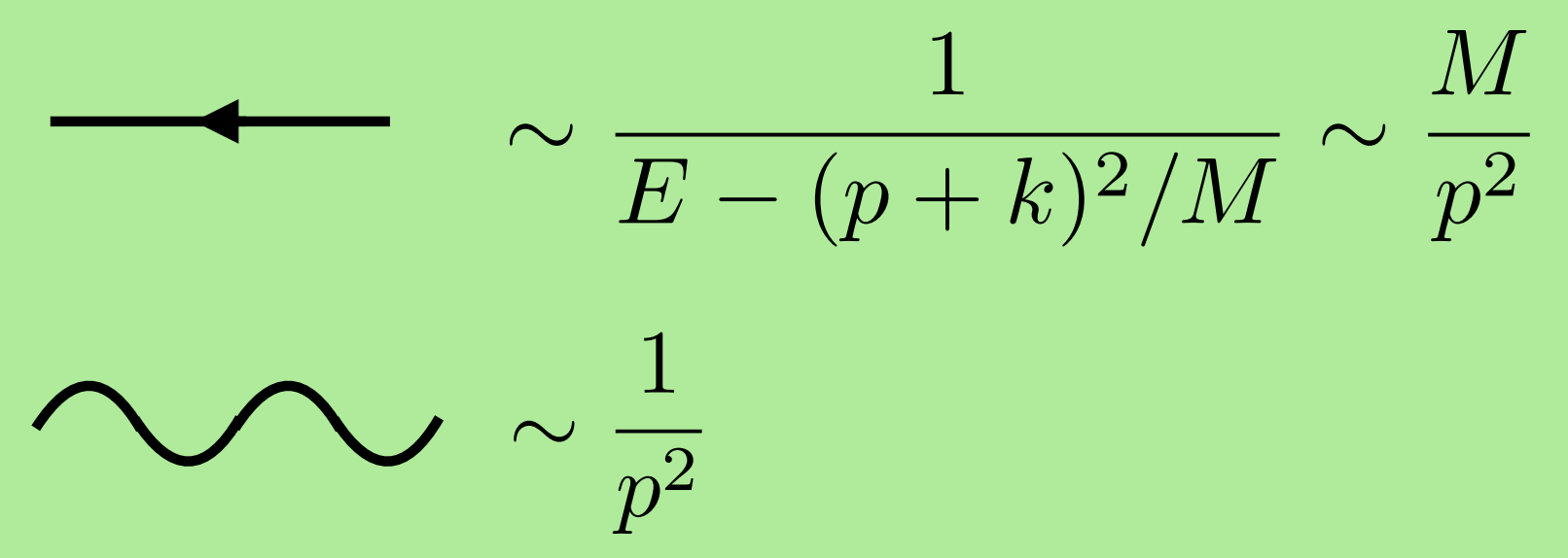

$$
\int d E \int d^{3} k \sim \frac{p^{5}}{M}
$$

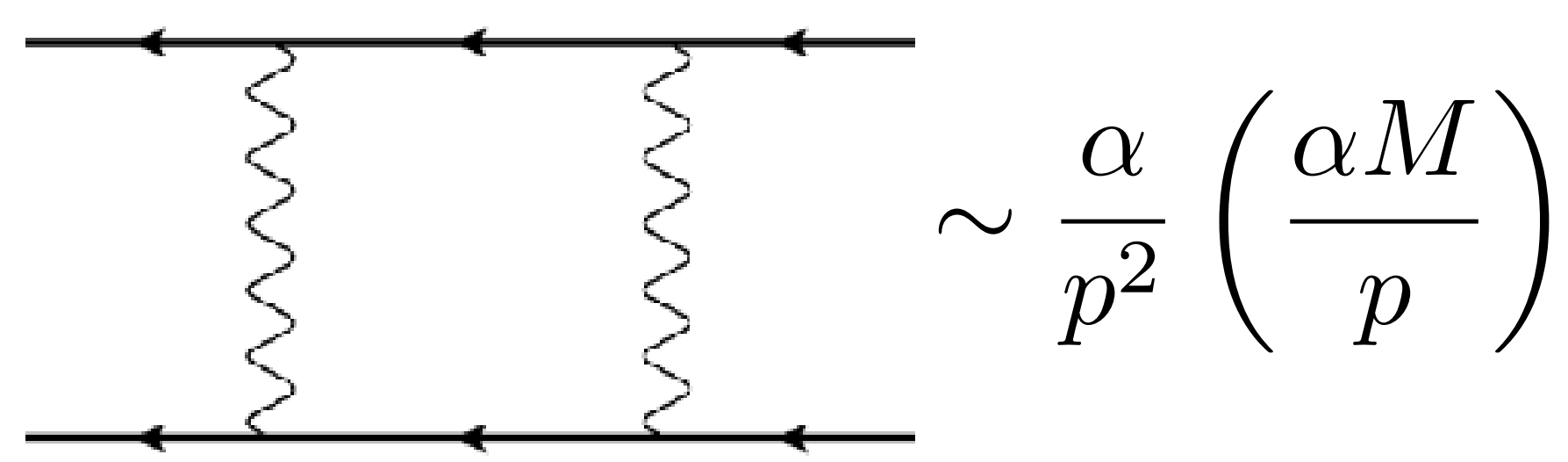




\section{Two Charged Particles in a Box}

In finite-volume with PBCs, momenta are quantized $\quad p=\frac{2 \pi}{L} n, n \in \mathbb{Z}^{3}$

Coulomb ladder diagrams must be nonperturbatively resumed for large enough volumes that $\alpha M / p \sim \alpha M L \gg 1$

— technically challenging unsolved problem

For intermediate volumes $\frac{1}{m_{\pi}} \ll L \ll \frac{1}{\alpha M}$ Coulomb effects are perturbative

Beane and Savage derived generalized Lüscher quantization condition relating FV energy shifts and scattering phase shifts accurate to $\mathcal{O}(\alpha), \mathcal{O}(\alpha M L)$

$$
C_{\eta(p)}^{2} p \cot \delta+\alpha M h(\eta)=-\frac{1}{a_{C}}+\frac{1}{2} r_{0} p^{2}+\ldots=\frac{1}{\pi L} \mathcal{S}^{C}\left(\frac{p L}{2 \pi}\right)+\alpha M\left[\ln \left(\frac{4 \pi}{\alpha M L}\right)-\gamma_{E}\right]
$$

Beane and Savage, PRD 90 (2014)

Range of validity of Beane-Savage regime needs to be explored in LQCD+QED $L$ 


\section{Lattice QCD+QED}

Dynamical QCD + QED ensembles generated by CSSM/QCDSF/UKQCD collaborations
$\alpha=0.1$

$$
a=0.068(2) \mathrm{fm}
$$$$
m_{\bar{K}^{0}}=404(1)(12) \mathrm{MeV}
$$$$
a L=32,48
$$

$N_{f}=2+1$ quark flavors with masses tuned to symmetric point where all neutral pseudoscalar mesons are degenerate, $m_{\bar{u} u} \approx m_{\bar{d} d}=m_{\bar{s} s}$

Quark mass tuning removes strong isospin breaking between $\pi^{+}$and $\bar{K}^{0}$ masses, remaining mass difference pure QED

QED effects explored by comparing systems of $1-12 \pi^{+}$mesons with systems of 1-12 $\bar{K}^{0}$ mesons 


\section{Charged Hadron Masses}

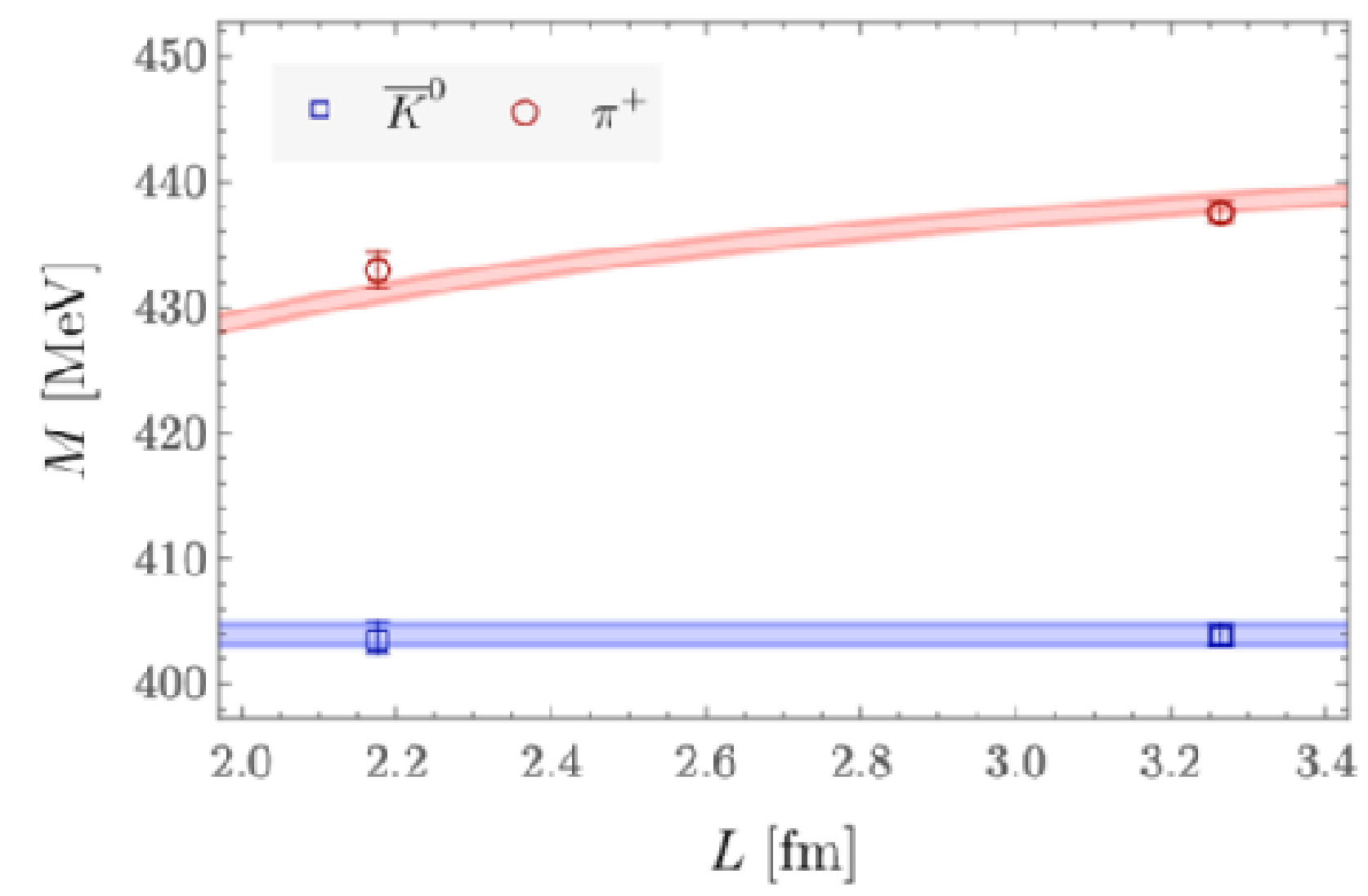

FV effects on $\pi^{+}$mass described by NRQED $D_{L}$ at NLO (1 fit parameter) for $\mathrm{L}=32,48$

$e^{-m L} \mathrm{FV}$ effects clearly present at $\mathrm{L}=24$ for $\pi^{+}$and $\bar{K}^{0}$, volume excluded from analysis

Horsley et al, J. Phys. G 43 (2016)

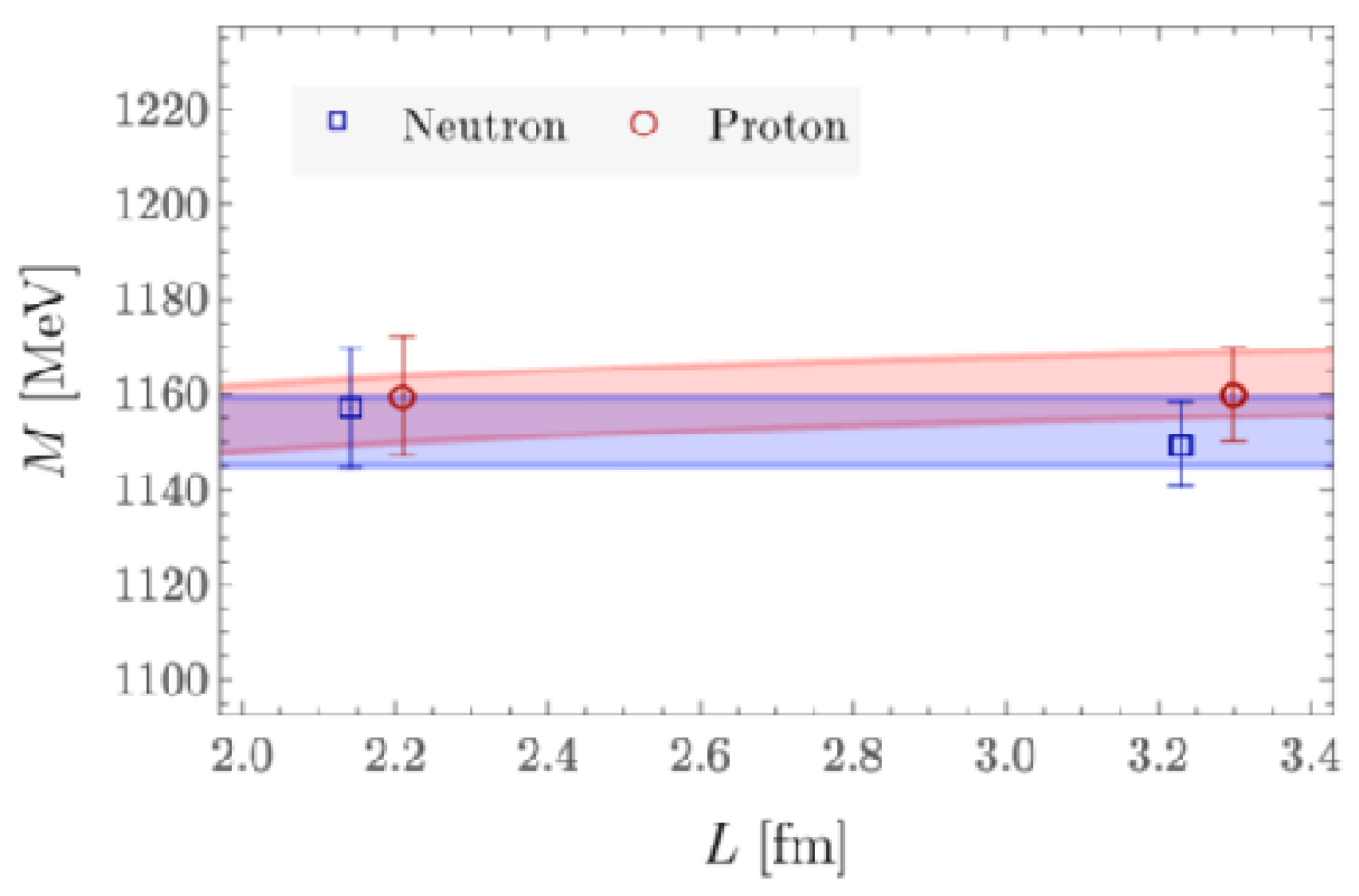

FV effects on proton mass described by NRQED ${ }_{L}$, suppressed compared to pion

$$
\alpha /\left(M_{p} L\right) \ll \alpha /\left(m_{\pi^{+}} L\right)
$$

$e^{-m L} \mathrm{FV}$ effects on neutron mass appear negligible 


\section{Many Charged Mesons}

Correlation functions for up to $\mathrm{N}=12$ charged pions can be computed from one pair of point-to-all quark propagators, as in previous NPLQCD studies without QED

Allows study of large charge densities: $\quad Z \alpha \leq 1.2 \quad \frac{Z \alpha}{L^{3}} \lesssim 0.12 \mathrm{fm}^{-3}$

Thermal effects become increasingly important for larger particle number

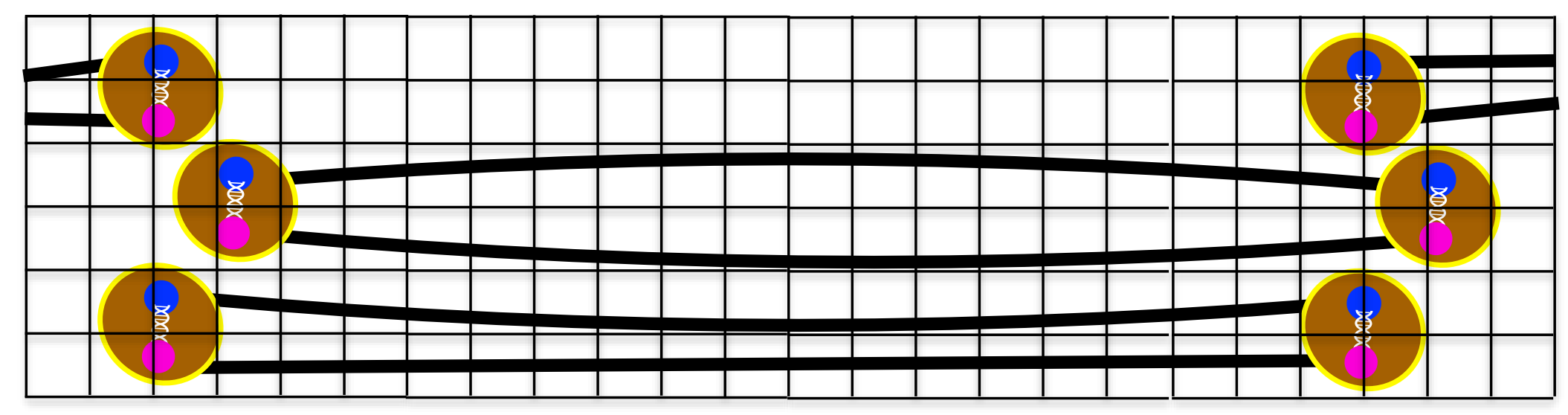

Fit results for $\mathrm{k}<\mathrm{N}$ particles used to determine thermal effects for $\mathrm{N}$ particles

$$
\begin{aligned}
G(N, t)= & \sum_{n} Z_{n}\left(e^{-E_{n}(N) t}+e^{-E_{n}(N)(\beta-t)}\right) \\
& +\sum_{k=1}^{[n / 2]} \widetilde{Z}_{0}^{k}\left(e^{-E_{0}(k) t} e^{-E_{0}(N-k)(\beta-t)}+e^{-E_{0}(k)(\beta-t)} e^{-E_{0}(N-k) t}+\ldots\right)
\end{aligned}
$$




\section{Many Fits}

Wide range of possible fit ranges sampled using automated procedure

Weighted average of all fits determines final results
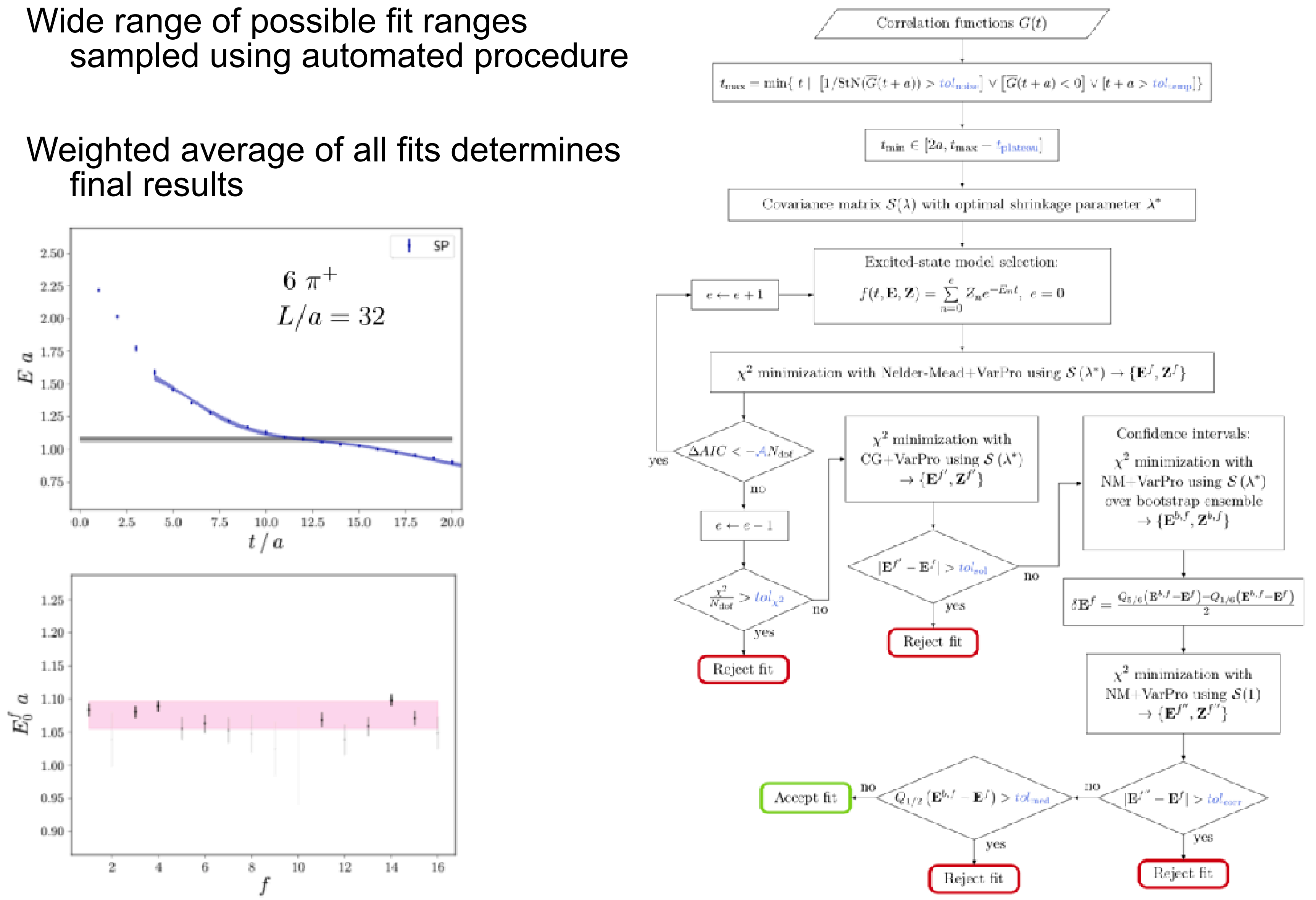


\section{Charged Hadron Interactions}
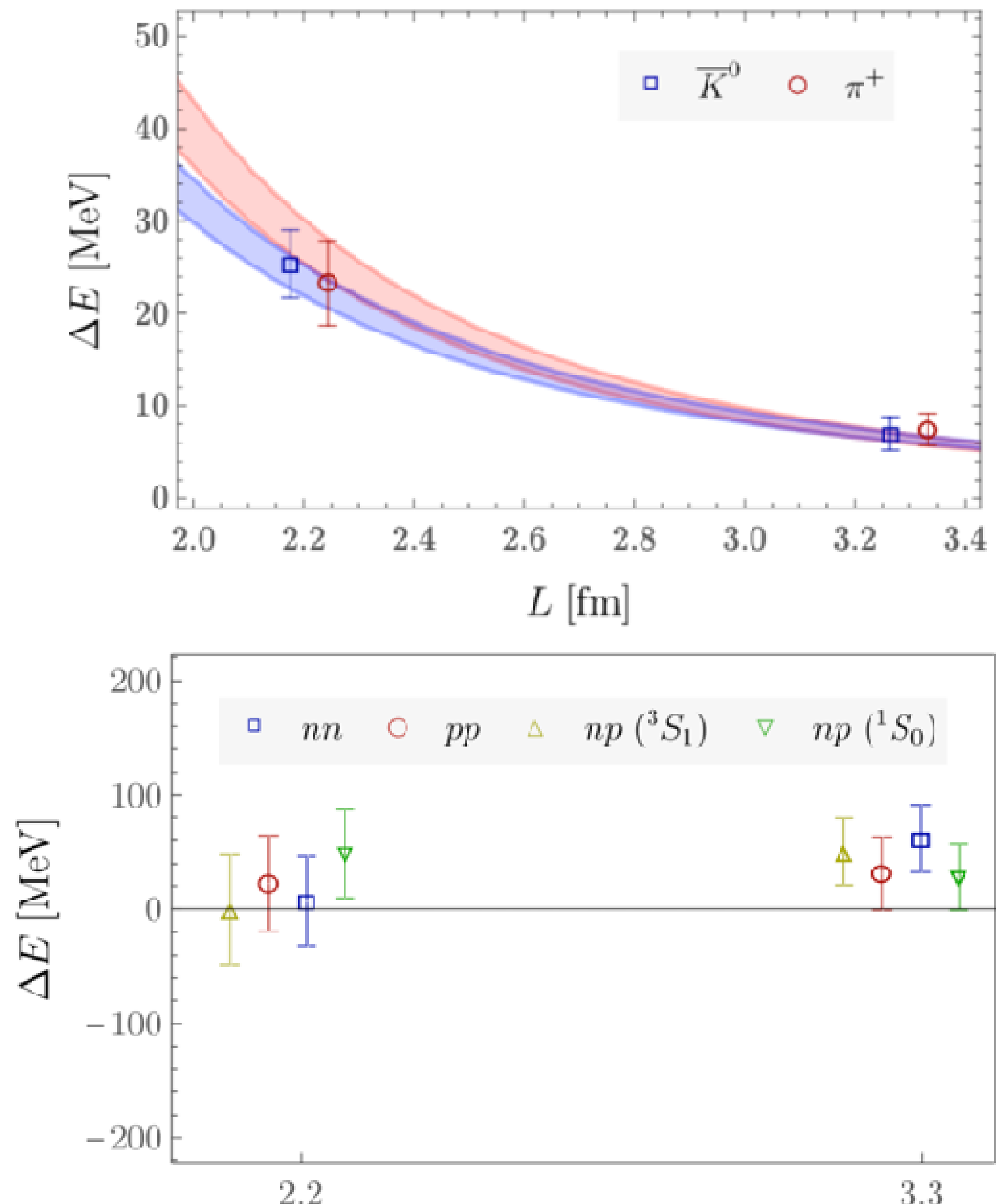

$L[\mathrm{fm}]$
Coulomb effects on $\pi^{+} \pi^{+} \mathrm{FV}$ energy shift might be expected to be large since

$$
\alpha m_{\pi^{+}} L \sim 0.50,0.74
$$

QED effects $<10 \%$ of total shift, even at largest volume

$\Delta E_{\pi^{+} \pi^{+}}=7.4(1.7) \mathrm{MeV}$

$\Delta E_{\pi^{+} \pi^{+}}-\Delta E_{K^{0} K^{0}}=0.26(58) \mathrm{MeV}$

Nucleon-nucleon systems have stronger Coulomb effects, still not resolved despite

$\alpha M_{p} L \sim 1.3,1.9$

Is this consistent with NRQED ${ }_{L}$ ? 


\section{Nonlocality}

Details of NRQED $L_{L}$ must be understood to interpret $L Q C D+Q E D_{L}$ results in EFT

Significant recent progress in understanding NRQED $D_{L}$ subtleties for single hadrons

Davoudi and Savage, PRD 90 (2014) Borsanyi et al, Science, vol. 347 (2015) Fodor et al, Phys. Lett. B 755 (2016)

Lee and Tiburzi, PRD 93 (2016) Matzelle and Tiburzi, PRD 95 (2017)

Davoudi, Harrison, Jüttner, Portelli, and Savage, PRD 99 (2019)

- locality violation from zero-mode subtraction allows IR scales such as $L$ to appear in coefficients of UV divergences and renormalized couplings

— careful matching between $\mathrm{QED}_{L}$ and $\mathrm{NRQED} D_{L}$ allows nonlocal counterterms to be determined
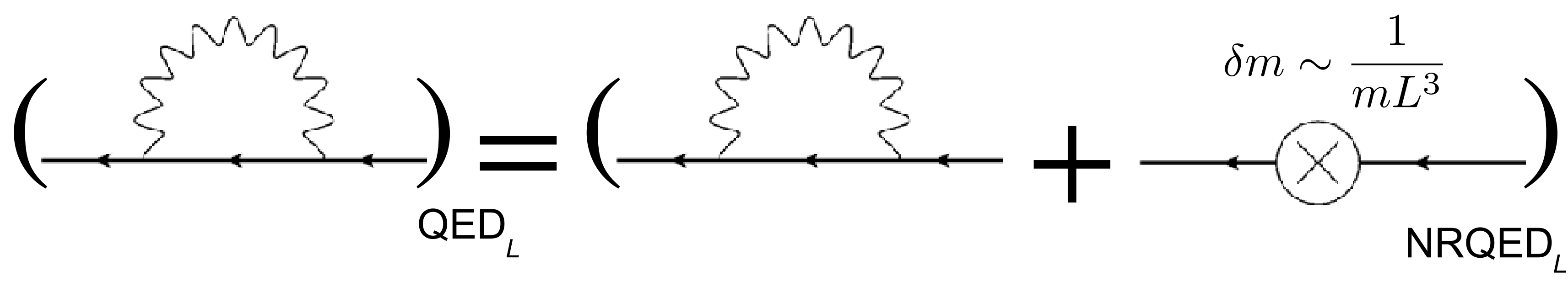

Remaining questions for multi-particle systems:

How do non-local counterterms modify 2-body interactions?

Are non-local 2-body effects enhanced by Coulomb ladder diagrams? 


\section{Matching NRQED $D_{L}$ and QED $_{L}$}

Tree-level $\mathrm{QED}_{L} 2$-> 2 amplitude reproduced by $\mathrm{NRQED}_{L}$ with $\quad \psi=\sqrt{2 M} e^{i M t} \varphi$

$\mathcal{L}^{\mathrm{QFFI}_{L}}=-\left(D_{\mu} \varphi\right)^{\dagger} D^{\mu} \varphi-M^{2} \varphi^{\dagger} \varphi-8 \pi a M\left(\varphi^{\dagger} \varphi\right)^{2}+\mathcal{L}_{\gamma}^{\xi}$

$\mathcal{L}^{\mathrm{NRQED}_{L}}=\psi^{\dagger}\left(i D_{0}-\frac{D_{i} D^{i}}{2 M}\right) \psi-\frac{2 \pi a}{M}\left(\psi^{\dagger} \psi\right)^{2}+\mathcal{L}_{\gamma}^{\xi}$

One-level QED $L 2$-> 2 amplitude includes antiparticle pole contributions not present in NRQED $L$

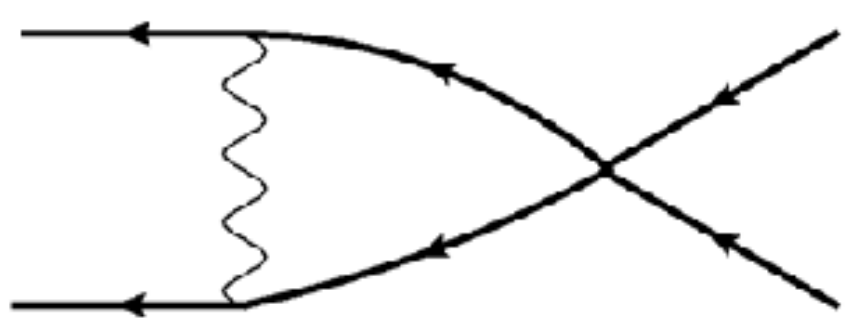

$\mathrm{QED}_{L}$

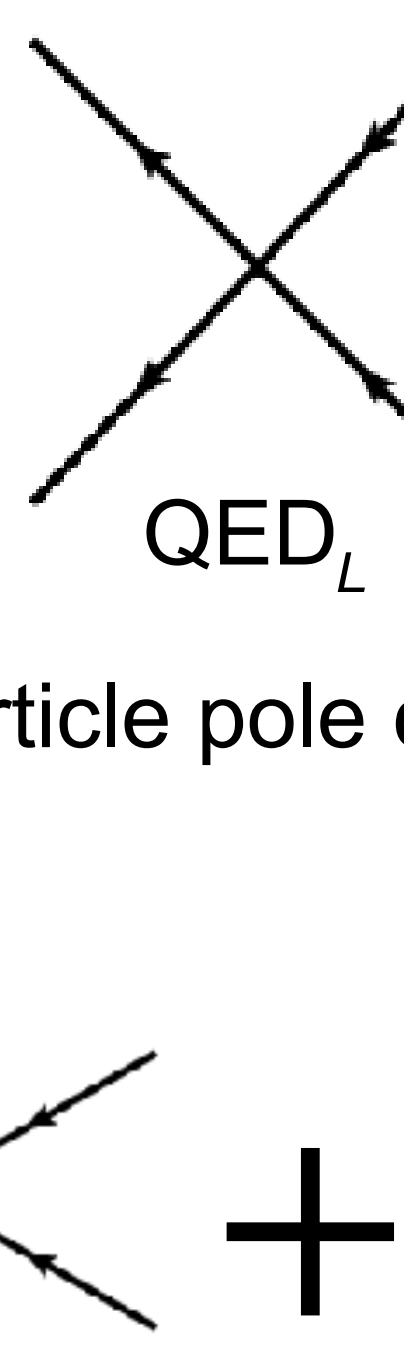
NRQED

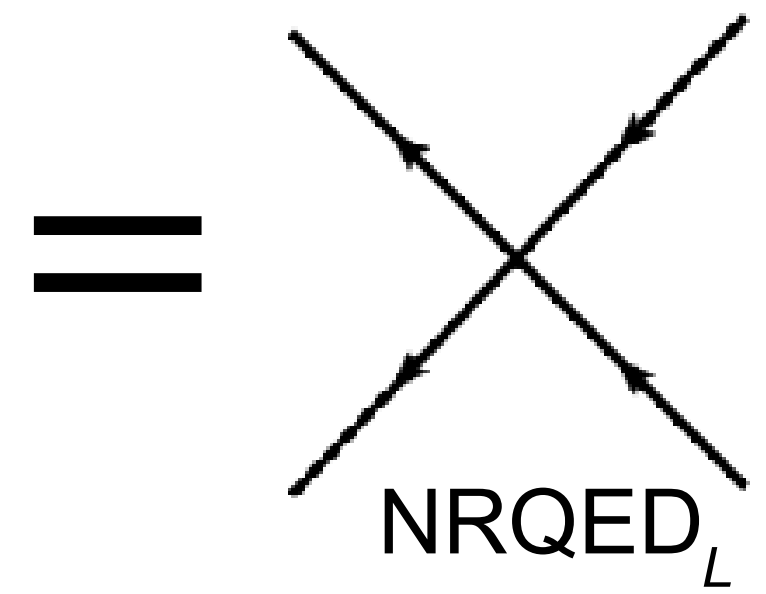

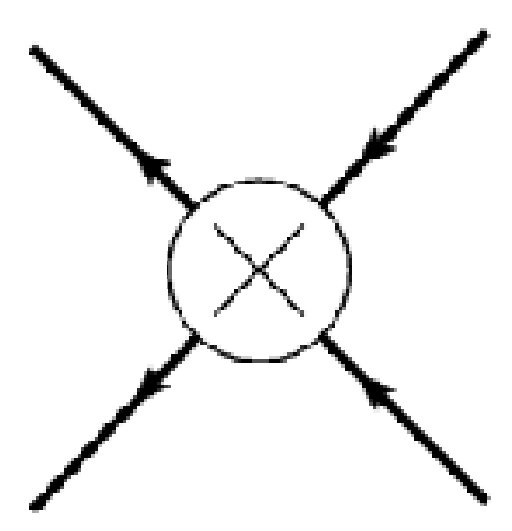

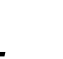

Explicit calculation shows antiparticle pole contribution suppressed by $\frac{\alpha}{(M L)^{3}}$

Antiparticle poles in Coulomb ladder diagrams further suppressed, nonlocal quartic interactions in NRQED $L$ only arise as high-order relativistic corrections 


\section{Matching $\mathrm{pNRQED} \mathrm{D}_{L}$ and $\mathrm{NRQED}_{L}$}

Dominant contributions arise from diagrams with Coulomb photon exchange between space-like separated particles

All nonlocal effects suppressed by powers of $1 /(M L)$

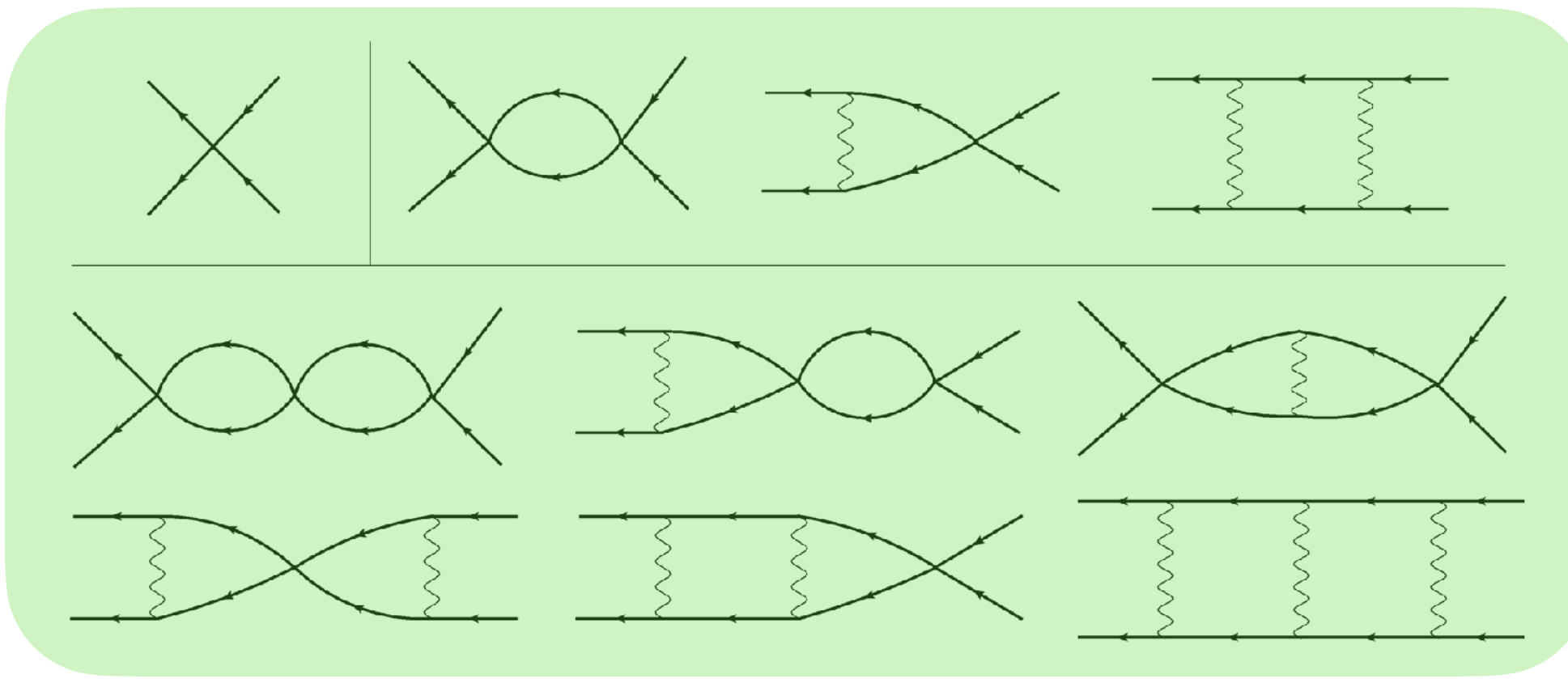

Contributions from radiation photon diagrams suppressed by $1 /(M L)$

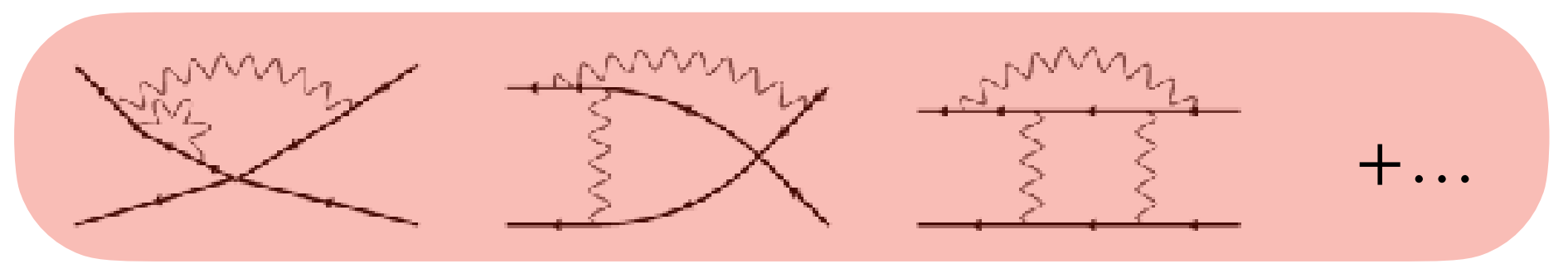

Coulomb photon contributions reproduced in Rayleigh-Schrödinger perturbation theory with potential

$$
\left\langle p_{1}^{\prime}, p_{2}^{\prime}|V| p_{1}, p_{2}\right\rangle=\left[\left(\frac{4 \pi a}{M L^{3}}\right)+\frac{\alpha}{\pi L} \sum_{i \in \mathbb{Z}^{3}}{ }^{\prime} \frac{1}{|i|^{2}}\right] \delta_{p_{1}+p_{2}-p_{1}^{\prime}-p_{2}^{\prime}}
$$

Defines leading-order potential NRQED $\left(\right.$ pNRQED $\left._{L}\right)$ accurate up to $1 /(M L)$ effects, higher-order potential computable as in infinite-volume pNRQED/pNRQCD 


\section{Many Charged Particles in a Box}

$3+$ particle systems achieve higher charge density in fixed volume, eventually probe nonperturbative relativistic QED effects

- comparison of $L Q C D+Q E D_{L}$ and $N R Q E D_{L}$ for multi-pion systems provides strong tests of validity of NRQED $L$

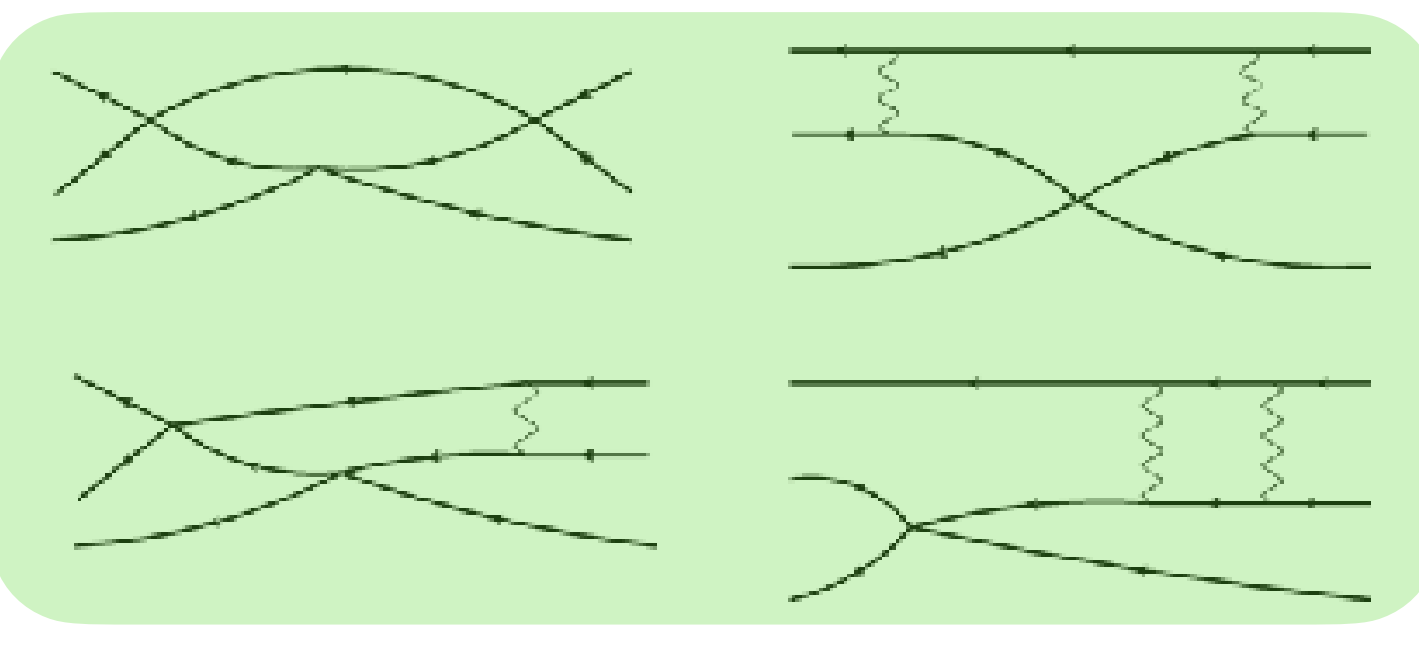

Coulomb photon contributions accessible to $\mathrm{pNRQED} \mathrm{D}_{L}$ dominant

$$
\begin{aligned}
H_{\text {int }}=- & -\sum_{\mathbf{k}} \widetilde{\psi}_{\mathbf{k}}^{\dagger}\left(\frac{\mathbf{k}^{4}}{8 M^{3}}\right) \widetilde{\psi}_{\mathbf{k}}+\frac{1}{2 L^{3}} \sum_{\mathbf{p}^{\prime}, \mathbf{p}, \mathbf{Q}} V\left(\mathbf{p}, \mathbf{p}^{\prime}\right) \widetilde{\psi}_{\mathbf{Q}-\mathbf{p}^{\prime}}^{\dagger} \widetilde{\psi}_{\mathbf{Q}+\mathbf{p}^{\prime}}^{\dagger} \widetilde{\psi}_{\mathbf{Q}-\mathbf{p}} \widetilde{\psi}_{\mathbf{Q}+\mathbf{p}} \\
& +\frac{\eta_{3}(\mu)}{(3 !) L^{6}} \sum_{\mathbf{Q}, \mathbf{p}, \mathbf{p}^{\prime}, \mathbf{q}, \mathbf{q}^{\prime}} \widetilde{\psi}_{\mathbf{Q}+\mathbf{p}^{\prime}}^{\dagger} \widetilde{\psi}_{\mathbf{Q}+\mathbf{q}^{\prime}}^{\dagger} \widetilde{\psi}_{\mathbf{Q}-\mathbf{p}^{\prime}-\mathbf{q}^{\prime}}^{\dagger} \widetilde{\psi}_{\mathbf{Q}+\mathbf{p}} \widetilde{\psi}_{\mathbf{Q}+\mathbf{q}} \widetilde{\psi}_{\mathbf{Q}-\mathbf{p}-\mathbf{q}}
\end{aligned}
$$

Radiation photon diagrams again suppressed by $1 /(M L)$
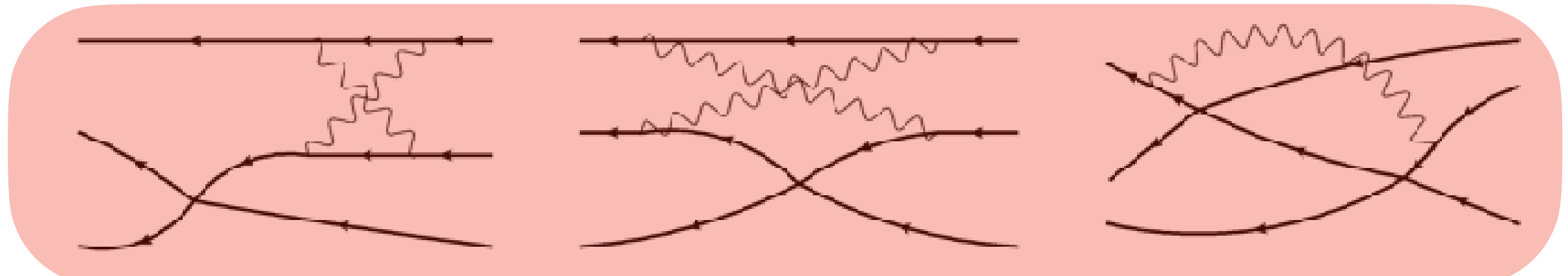

$\mathrm{pNRQED}_{L}$ suitable for calculations of many-pion energy shifts up to $1 /(M L)$ effects 


\section{Relativistic Corrections}

Relativistic corrections to NRQED also arise at higher order

$$
\mathcal{L}=\psi^{\dagger}\left(i D_{0}-\frac{\mathbf{D}^{2}}{2 M}\right) \psi-\frac{1}{2}\left(\frac{4 \pi a}{M}\right)\left(\psi^{\dagger} \psi\right)^{2}-\frac{\eta_{3}(\mu)}{3 !}\left(\psi^{\dagger} \psi\right)^{3}+\mathcal{L}_{\gamma}^{\xi}+\mathcal{L}_{r} .
$$

LECs for relativistic corrections can be obtained by matching threshold expansion of quantization condition (without QED)

Hansen and Sharpe, PRD 93 (2016)

$\mathcal{L}_{r}=\psi^{\dagger}\left(\frac{\mathbf{D}^{4}}{8 M^{3}}\right) \psi-\frac{1}{2}\left(\frac{\pi a}{M}\right)\left(a r-\frac{1}{M^{2}}\right)\left(\psi^{\dagger} \psi\right)\left(\psi^{\dagger} \mathbf{D}^{2} \psi+\psi \mathbf{D}^{2} \psi^{\dagger}\right)$

Matching on to potential NRQED $D_{L}$ gives

$$
V\left(\mathbf{p}^{\prime}, \mathbf{p}\right)=\frac{4 \pi}{M}\left(a+\frac{a}{4}\left(a r-\frac{1}{M^{2}}\right)\left(\mathbf{p}^{2}+\mathbf{p}^{\prime 2}\right)+\ldots\right)+\frac{4 \pi \alpha}{\left|\mathbf{p}^{\prime}-\mathbf{p}\right|^{2}}\left(1-\delta_{\mathbf{p}, \mathbf{p}^{\prime}}\right)
$$




\section{FV Energy Shifts in pNRQED $D_{L}$}

$n$-boson energy shifts given by Rayleigh-Schrödinger perturbation theory

$$
\left|\mathbf{p}_{1}, \ldots, \mathbf{p}_{n}\right\rangle=\frac{1}{\sqrt{n}} \widetilde{\psi}_{\mathbf{p}_{1}}^{\dagger} \times \ldots \times \widetilde{\psi}_{\mathbf{p}_{2}}^{\dagger}|0\rangle \quad\left\langle\mathbf{0}, \ldots \mathbf{0}\left|H_{\text {int }}\right| \mathbf{0}, \ldots \mathbf{0}\right\rangle=\frac{1}{L^{3}}\left(\begin{array}{l}
n \\
2
\end{array}\right) V(\mathbf{0}, \mathbf{0})+\frac{1}{L^{6}}\left(\begin{array}{l}
n \\
3
\end{array}\right) \eta_{3}(\mu) .
$$

Higher-orders, e.g. $\mathrm{N}^{3}$ LO in strong interactions + NLO in QED

$$
\begin{aligned}
\Delta E_{n}^{\mathrm{N}^{3} \mathrm{LO}, \mathrm{PC} 2}=\frac{4 \pi \bar{a}_{C}}{M L^{3}}\left(\begin{array}{l}
n \\
2
\end{array}\right)\{1 & -\left(\frac{\bar{a}_{C}}{\pi L}\right) \mathcal{I}+\left(\frac{\bar{a}_{C}}{\pi L}\right)^{2}\left[\mathcal{I}^{2}+(2 n-5) \mathcal{J}\right] \\
& \left.\quad-\left(\frac{\bar{a}_{C}}{\pi L}\right)^{3}\left[\mathcal{I}^{3}+(2 n-7) \mathcal{I} \mathcal{J}+\left(5 n^{2}-41 n+63\right) \mathcal{K}\right]\right\} \\
+ & \frac{4 \eta_{L}}{M L^{2}}\left(\begin{array}{l}
n \\
2
\end{array}\right)\left\{-\left(\frac{\bar{a}_{C}}{\pi L}\right)^{2} 2 \mathcal{J}-\left(\frac{\eta_{L}}{\pi^{2}}\right) \mathcal{K}\right. \\
& \left.+\left(\frac{\bar{a}_{C}}{\pi L}\right)^{2}\left[2 \mathcal{I} \mathcal{J}+\mathcal{R}_{22}+(4 n-10) \mathcal{K}-4 \pi^{4}\left[\ln \left(\eta_{L}\right)+\gamma_{E}\right]\right]\right\} \\
+ & \left(\begin{array}{c}
n \\
3
\end{array}\right) \frac{1}{L^{6}}\left[\eta_{3}(\mu)+\frac{64 \pi \bar{a}_{C}^{4}}{M}(3 \sqrt{3}-4 \pi) \ln (\mu L)-\frac{96 \bar{a}_{C}^{4}}{\pi^{2} M} \mathcal{S}_{\mathrm{MS}}\right] .
\end{aligned}
$$

Effective range and relativistic corrections act as modification to scattering length

$$
a_{C}=\bar{a}_{C}(L)-\frac{2 \pi \bar{a}_{C}(L)^{2}}{L^{3}}\left(\bar{a}_{C}(L) r-\frac{1}{2 M^{2}}\right)
$$

$\begin{array}{ll}\text { Extends: } & \text { Huang and Yang, Phys. Rev. } 105 \text { (1957) } \\ & \text { Lüscher, Commun. Math. Phys. } 105 \text { (1986) }\end{array}$

Beane, Detmold, and Savage, PRD 76 (2007)

Beane and Savage, PRD 90 (2014)

Hansen and Sharpe, PRD 93 (2016) 


\section{FV Energy Shifts in pNRQED $D_{L}$}

$$
\begin{aligned}
\Delta E_{n}^{\mathrm{N}^{3} \mathrm{LO}, \mathrm{PC} 2}= & \frac{4 \pi \bar{a}_{C}}{M L^{3}}\left(\begin{array}{c}
n \\
2
\end{array}\right)\left\{1-\left(\frac{\bar{a}_{C}}{\pi L}\right) \mathcal{I}+\left(\frac{\bar{a}_{C}}{\pi L}\right)^{2}\left[\mathcal{I}^{2}+(2 n-5) \mathcal{J}\right]-\left(\frac{\bar{a}_{C}}{\pi L}\right)^{3}\left[\mathcal{I}^{3}+(2 n-7) \mathcal{I} \mathcal{J}+\left(5 n^{2}-41 n+63\right) \mathcal{K}\right]\right\} \\
& +\frac{4 \eta_{L}}{M L^{2}}\left(\begin{array}{c}
n \\
2
\end{array}\right)\left\{-\left(\frac{\bar{a}_{C}}{\pi L}\right) 2 \mathcal{J}-\left(\frac{\eta_{L}}{\pi^{2}}\right) \mathcal{K}+\left(\frac{\bar{a}_{C}}{\pi L}\right)^{2}\left[2 \mathcal{I} \mathcal{J}+\mathcal{R}_{22}+(4 n-10) \mathcal{K}-4 \pi^{4}\left[\ln \left(\eta_{L}\right)+\gamma_{E}\right]\right]\right\} \\
& +\left(\begin{array}{l}
n \\
3
\end{array}\right) \frac{1}{L^{6}}\left[\eta_{3}(\mu)+\frac{64 \pi \bar{a}_{C}^{4}}{M}(3 \sqrt{3}-4 \pi) \ln (\mu L)-\frac{96 \bar{a}_{C}^{4}}{\pi^{2} M} \mathcal{S}_{\mathrm{MS}}\right] .
\end{aligned}
$$

\section{Important features:}

2-body log involves FV analog of Coulomb expansion parameter $\eta_{L}=\frac{\alpha M}{2 p}=\frac{\alpha M L}{4 \pi}$ Coulomb photon effects enter as power series in $\eta_{L}$ with $\mathrm{O}(1)$ coefficients FV Coulomb perturbative when $\frac{\alpha M L}{4 \pi} \ll 1$, including $\alpha M L \sim 1$

Physical quark mass

$$
\begin{aligned}
& \alpha M L \ll 1 \\
& \frac{\alpha M L}{4 \pi} \ll 1 \ll 2.1 \mathrm{fm} \\
&
\end{aligned}
$$$$
\text { protons with } \alpha=0.1
$$ 


\section{Power Counting}

With $m_{\pi^{+}}=449(1)(13) \mathrm{MeV}$ and volumes $L=2.2 \mathrm{fm}, 3.2 \mathrm{fm}$

$$
\frac{\alpha m_{\pi} L}{4 \pi}=0.04,0.06 \quad \frac{a_{\pi \pi}}{L}=0.10,0.07
$$

Hierarchy between QCD and QED effects volume dependent, two reasonable power counting for this range of volumes

$$
\text { PC1 } \frac{\alpha m_{\pi} L}{4 \pi} \sim \frac{a_{\pi \pi}}{L} \quad \text { PC2 } \quad \frac{\alpha m_{\pi} L}{4 \pi} \sim\left(\frac{a_{\pi \pi}}{L}\right)^{2}
$$

Results for PC1 computed to NNLO

Known higher-order results for pure QCD allow PC2 to be extend to $N^{3} L O$ 


\section{EFT Fits}

EFT parameters $a^{K^{0} K^{0}}, a_{C}^{\pi^{+} \pi+}$ and three-body couplings $\eta^{\bar{K}^{0} \bar{K}^{0} \bar{K}^{0}}, \eta^{\pi^{+} \pi^{+} \pi^{+}}$ determined from global fit to all FV shifts with 2-12 mesons and correlated differences $\Delta E_{N \pi^{+}}-\Delta E_{N K^{0}}$

NNLO fits with both power counting give $\chi^{2} /$ dof $\sim 1.3$

$\mathrm{N}^{3} \mathrm{LO}$ fits (pc 2) add three-body forces, better agreement $\quad \chi^{2} /$ dof $\sim 0.8$
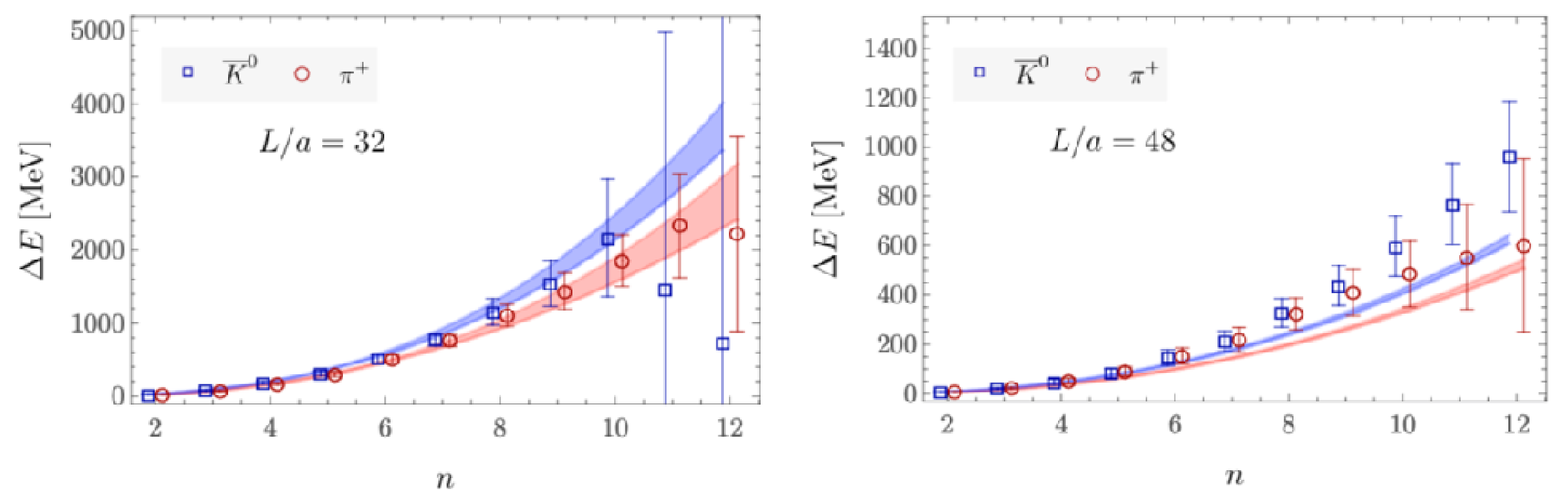


\section{EFT Fits}

QED effects resolved at $1-2 \sigma$ for systems of $3-12$ mesons

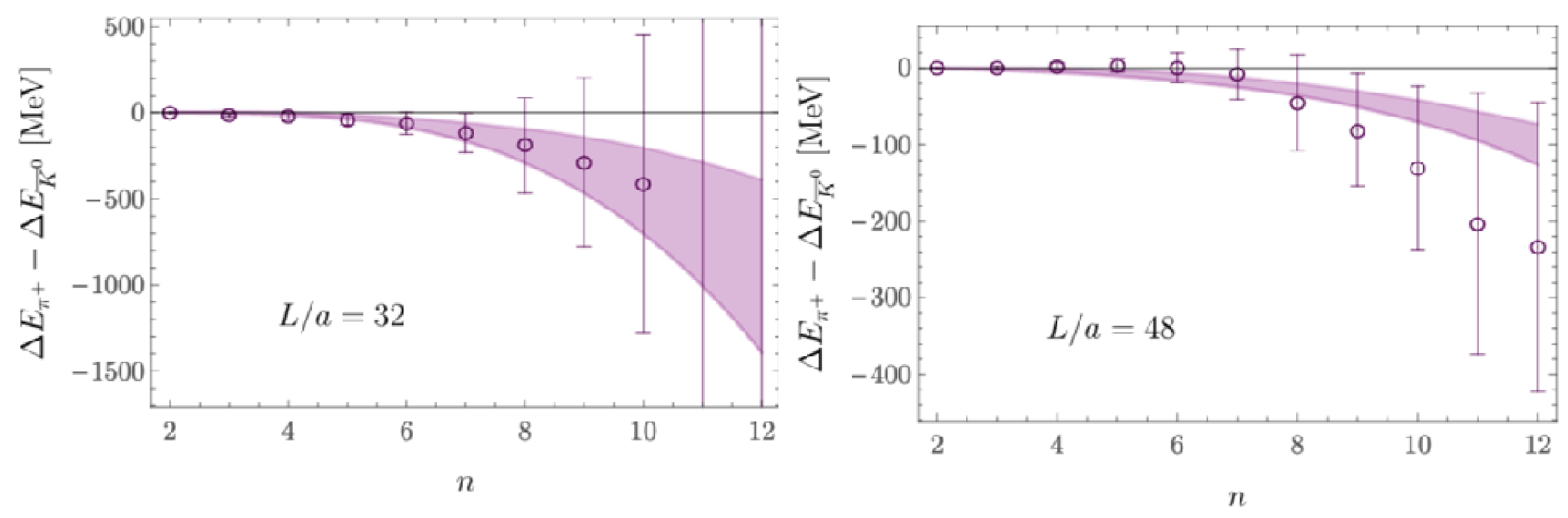

Negative sign arises from zero-mode subtraction, full QED effects reproduced by Coulomb effects in NRQED $L$ 


\section{EFT Results}

Coulomb scattering length for $\pi^{+} \pi^{+}$differs from $K^{0} K^{0}$ scattering length

$$
a^{\bar{K}^{0} \bar{K}^{0}} m_{\bar{K}^{0}}=0.337(19) \quad a_{C}^{\pi^{+} \pi^{+}} m_{\bar{K}^{0}}=0.464(29)
$$

Three-body forces clearly resolved, no QED effects visible on strength of threebody forces

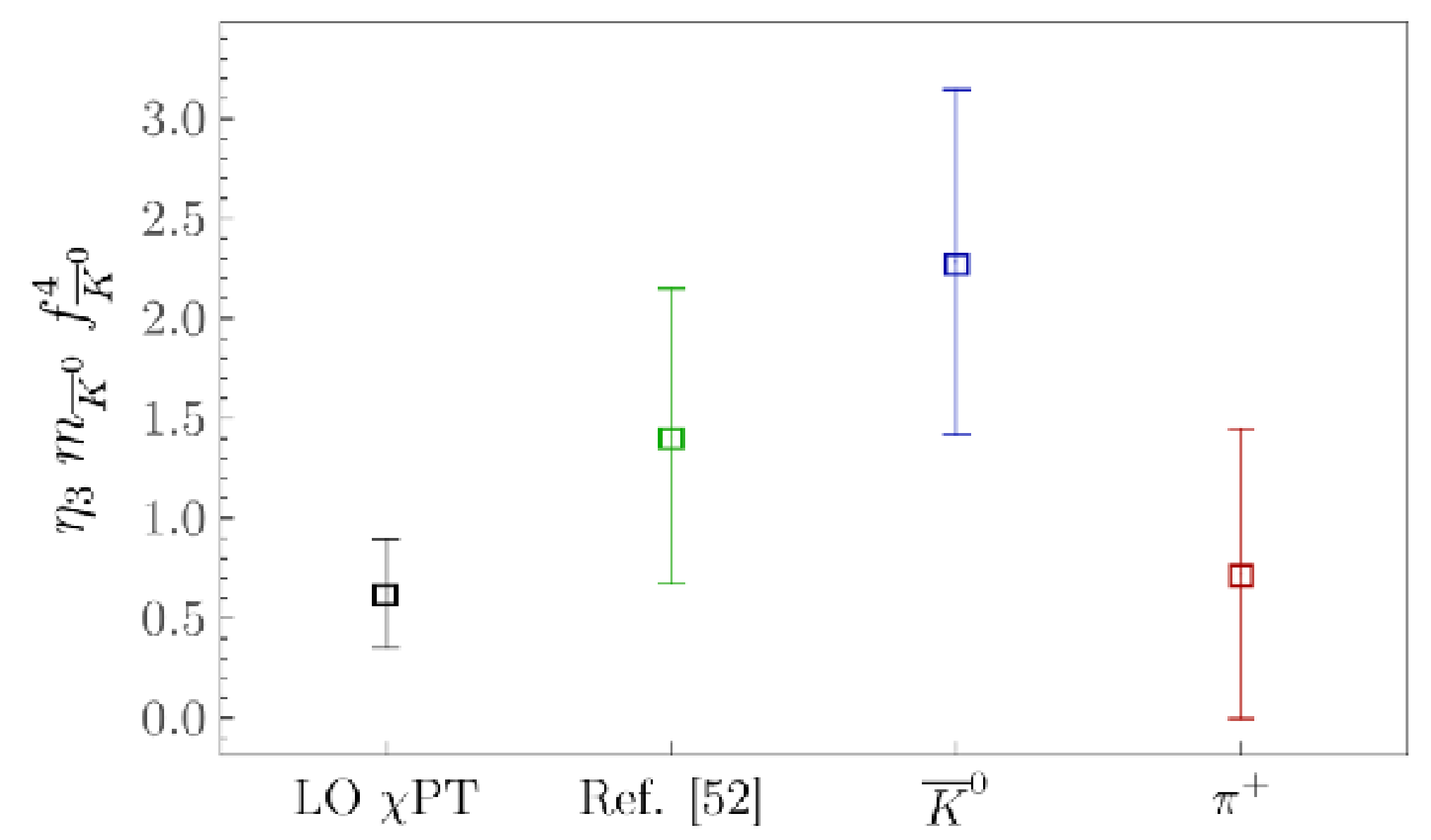

(all results valid for unphysical quark masses used in calculation) 


\section{Summary}

Nonlocal counterterms in NRQED are not enhanced by Coulomb ladders (at least at 2-loop order), appear only as relativistic corrections

FV effects on interacting charged hadron systems in LQCD+QED ${ }_{L}$ consistent with $\mathrm{NRQED}_{L}$ up to $Z \alpha \leq 1.2, Z \alpha / L^{3} \lesssim 0.12 \mathrm{fm}^{-3}$ at unphysically large quark mass

Size of QED effects consistent with Coulomb expansion parameter

$$
\eta_{L}=\frac{\alpha M}{2 p}=\frac{\alpha M L}{4 \pi} \ll \alpha M L
$$

NRQED $L$ with perturbative Coulomb should converge for proton-proton systems at the physical point with $L \ll 20 \mathrm{fm}$ 